SHEP-09-03

\title{
Neutral Higgs boson pair production in photon-photon annihilation in the Two Higgs Doublet Model
}

\author{
Abdesslam Arhrib ${ }^{1,2}$, Rachid Benbrik ${ }^{2,3,4}$, Chuan-Hung Chen $^{3,4}$ and Rui Santos ${ }^{5}$ \\ 1 Département de Mathématique, Faculté des Sciences and Techniques, \\ Université Abdelmalek Essâ̂di, B. 416, Tangier, Morocco. \\ ${ }^{2}$ LPHEA, Faculté des Sciences-Semlalia, B.P. 2390 Marrakesh, Morocco. \\ 3 Department of Physics, National Cheng Kung University, Taiwan 701, Taiwan. \\ 4 National Center for Theoretical Physics, Taiwan 701, Taiwan. and \\ 5 NExT Institute and School of Physics and Astronomy, \\ University of Southampton Highfield, Southampton SO17 1BJ, UK.
}

(Dated: October 30, 2018)

\begin{abstract}
We study double Higgs production in photon-photon collisions as a probe of the new dynamics of Higgs interactions in the framework of two Higgs Doublet Models. We analyze neutral Higgs bosons production and decay in the fusion processes, $\gamma \gamma \rightarrow S_{i} S_{j}, S_{i}=h^{0}, H^{0}, A^{0}$, and show that both $h^{0} h^{0}$ and $A^{0} A^{0}$ production can be enhanced by threshold effects in the region $E_{\gamma \gamma} \approx 2 m_{H \pm}$. Resonant effects due to the heavy Higgs, $H^{0}$, can also play a role in the cross section enhancement when it is allowed to decay to two light CP-even $h^{0}$ or to two light CP-odd $A^{0}$ scalars. We have scanned the allowed parameter space of the Two Higgs Doublet Model and found a vast region of the parameter space where the cross section is two orders of magnitude above the Standard Model cross section. We further show that the Standard Model experimental analysis can be used to discover or to constraint the two Higgs doublet model parameter space.
\end{abstract}

PACS numbers: 12.60.Fr, 14.80.Cp 


\section{INTRODUCTION}

The search for Higgs bosons is the prime task of CERN's Large Hadron Collider (LHC), with operation scheduled now for 2009. With the LHC guidance, the International $e^{+} e^{-}$ Linear Collider (ILC), which is currently being designed, will further improve our knowledge of the Higgs sector if that is how Nature decided to create mass. It was demonstrated in Ref. [1] that physics at the LHC and at the ILC will be complementary to each other in many respects. In many cases, the ILC can significantly improve the LHC measurements. If a Higgs boson is discovered, it will be crucial to determine its couplings with high accuracy, to understand the so-called mechanism of electroweak symmetry breaking [2]. The high resolution profile determination of a light Higgs boson (mass, couplings, self couplings, etc.) can be carried out at the ILC, where clear signals of Higgs events are expected with backgrounds that can be reduced to a manageable level. This is exactly the case of processes such as $e^{+} e^{-} \rightarrow \gamma \gamma \rightarrow S_{i} S_{j}$ where $S_{i}=h^{0}, H^{0}, A^{0}$. This fusion process can produce a Standard Model (SM) Higgs boson or one predicted by the various extensions of the SM, such as the Minimal Supersymmetric Standard Model (MSSM) or Two Higgs Doublet Models (2HDM).

According to its Reference Design Report [3], the ILC will run at an energy of $\sqrt{s}=500$ $G e V$ with a total luminosity of $\mathcal{L}=500 \mathrm{fb}^{-1}$ within the first four years of operation and $\mathcal{L}=1000 \mathrm{fb}^{-1}$ during the first phase of operation with $\sqrt{s}=500 \mathrm{GeV}$. An $e^{+} e^{-}$collider is uniquely capable of operation at a series of energies near the threshold of a new physics process. This is an extremely powerful tool for precision measurements of particle masses and unambiguous particle spin determination. Various ILC physics studies, indicate that a $\sqrt{s}=500 \mathrm{GeV}$ collider can have a great impact on understanding new physics at the $T e V$ scale. An energy upgrade up to $\sqrt{s} \sim 1 \mathrm{TeV}$ would probably open the doors to even greater discoveries. Another very unique feature of the ILC is that it can accommodate a $\gamma \gamma$ collider with the photon beams generated by using the Compton backscattering of the initial electron and laser beams [4]. In this case, the energy and luminosity of the photon beams would be of the same order of magnitude of the original electron beams. As the set of final states at a photon collider is much richer than that in the $e^{+} e^{-}$mode, it would open a wider window to probe new physics beyond the SM.

Since photons couple directly to all fundamental fields carrying electromagnetic charge, $\gamma \gamma$ collisions provide a comprehensive means of exploring virtual aspect of the SM and its 
extensions [5]. The production mechanism in hadron and $e^{+} e^{-}$machines are often more complex and model-dependent. Thus, a $\gamma \gamma$ collider is much more sensitive to new physics even at higher mass scales [6].

The primary mechanism of neutral Higgs boson production in $\gamma \gamma$ collisions is $\gamma \gamma \rightarrow$ $\left(h^{0}, H^{0}, A^{0}\right)$ [7, 8, 9, 10], but in order to explore the triple and quartic Higgs couplings at future high energy colliders, it is necessary to study the Higgs boson pair production process. The triple Higgs couplings of the 2HDM have been extensively studied at $e^{+} e^{-}$ linear colliders [11] and shown to provide an opportunity to measure those couplings. At photon-photon colliders, the cross section for neutral Higgs boson pair production has been calculated in [12, 13] in the SM and found to be rather small. In the 2HDM, the process $\gamma \gamma \rightarrow h^{0} h^{0}$ has been computed in the decoupling limit in [14, 15]. They found that the cross section can be substantially enhanced in the 2HDM and that the number of events expected at the Photon Collider will allow a determination or exclusion of some of the parameter space in the $2 \mathrm{HDM}$ potential.

In the MSSM, various studies for Higgs pair production at a photon collider have been performed. The process $\gamma \gamma \rightarrow h^{0} h^{0}$ was studied in [16] while reactions $\gamma \gamma \rightarrow$ $h^{0} H^{0}, h^{0} A^{0}, H^{0} H^{0}, H^{0} A^{0}$ were determined in [17]. Process $\gamma \gamma \rightarrow A^{0} A^{0}$ was calculated for the MSSM [18, 19] and shown to have a cross section of the order of $0.1-0.2 f b$ for a vast range of the photon-photon center of mass energy.

In this paper, we present a complete calculation of pair production of all neutral Higgs bosons at the one loop level in the 2HDM. We study the Higgs self couplings effects on the $\gamma \gamma \rightarrow h^{0} h^{0}$ and $\gamma \gamma \rightarrow A^{0} A^{0}$ cross sections and briefly comment on the $\gamma \gamma \rightarrow h^{0} A^{0}$, $\gamma \gamma \rightarrow h^{0} H^{0}, \gamma \gamma \rightarrow H^{0} A^{0}$ and $\gamma \gamma \rightarrow H^{0} H^{0}$ production modes. This exhausts all possible neutral scalar production processes in the 2HDM. A measurement of these processes can shed some light on the 2HDM triple Higgs couplings. However, even if the situation regarding a measurement of the vertex is not clear because no peak is detected, a vast region of the 2HDM parameter space will be excluded. The scalars will be detected via similar final states because both the $h^{0}$ and the $A^{0}$, when not too heavy, decay predominantly into fermions. In this regard, the knowledge of their exact total cross section and angular distributions may be helpful in order to distinguish between CP-even and CP-odd scalars. Moreover, it is well-known that in the 2HDM, both the CP-even $h^{0}$ and the CP-odd pseudo-scalar $A^{0}$ can be rather light [20]. In fact, the bounds on the $h^{0}$ and $A^{0}$ masses originate from 
the $e^{+} e^{-} \rightarrow h^{0} Z$ and $e^{+} e^{-} \rightarrow h^{0} A^{0}$ production processes with the Higgs decaying to some combination of jets (mainly $b$ jets) and $\tau$ leptons. The production process $e^{+} e^{-} \rightarrow h^{0} Z$ is proportional to $\sin ^{2}(\alpha-\beta)$ and this is the reason why LEP does not limit the mass of a light Higgs $h^{0}$ for $\sin (\alpha-\beta)=0.1$. For $\sin (\alpha-\beta)=0.3$ the bound is of the order of 80

$\mathrm{GeV}$ [21]. The pseudo-scalar mass is only limited by the results on $e^{+} e^{-} \rightarrow h^{0} A^{0}$. However, if the sum of the masses is above the LEP energy limit, again no bound applies.

A very interesting feature of $\gamma \gamma \rightarrow A^{0} A^{0}$ is that a light $A^{0}$ can easily emerge in the Nextto Minimal Supersymmetric Standard Model (NMSSM) and therefore comparison between models will certainly prove useful. In addition, we also take into account in our calculation the perturbativity, unitarity as well as vacuum stability constraints on the various parameters in the Higgs potential. We will show that after imposing those constraints, cross sections are still large enough, in the hundred of fempto-barn $(f b)$ region in some cases, to probe the $2 \mathrm{HDM}$ scalar sector. We will also study some of these processes in the decoupling limit and in the fermiophobic limit of the so-called type-I 2HDM.

The paper is organized as follows. In the next section, we review the 2HDM potential we will be using, give the analytical expressions for the triple and quartic Higgs couplings and list the theoretical constraints on the 2HDM scalar potential such as unitarity and vacuum stability. In Section III, we evaluate the double Higgs production cross section, $\gamma \gamma \rightarrow S_{i} S_{j}$ with $S_{i, j}=H^{0}, h^{0}, A^{0}$, in the general 2HDM paying special attention to $\gamma \gamma \rightarrow h^{0} h^{0}$ and $\gamma \gamma \rightarrow A^{0} A^{0}$. We then proceed to Section [V] where we present our numerical results for the general 2HDM and for two limiting cases: the decoupling limit and the fermiophobic limit. In Section $\nabla$ we discuss the final states in the different $2 \mathrm{HDM}$ scenarios. Our findings are summarized in Section VI.

\section{REVIEW OF THE TWO HIGGS DOUBLET MODEL}

\section{A. The Two Higgs doublet model}

Two Higgs doublet models are some of the most well studied extensions of the Standard Model. Various motivations for adding a second Higgs doublet to the Standard Model have been advocated in the literature [22, 23]. There are several types of 2HDM. While the coupling to gauge bosons is universal, there are many ways to couple the Higgs doublets to 
matter fields. Assuming natural flavor conservation [24] there are four ways to couple the Higgs to the fermions [25]. The most popular models are the type-I and the type-II models, denoted by 2HDM-I and 2HDM-II, respectively. In 2HDM-I, the quarks and leptons couple only to one of the two Higgs doublet which is exactly what happens in the SM. In 2HDM-II, one of the 2HDM fields couples only to down-type fermions (down-type quarks and charged leptons) and the other one only couples to up-type fermions in order to avoid the problem of flavor-changing neutral currents (FCNC's) at tree-level. There are two additional models less discussed in the literature: model III in which one of the doublets couples to all quarks and the other couples to all leptons and the type IV model is instead built such that one doublet couples to up-type quarks and to leptons and the other couples to down-type quarks. There is a class of models sometimes called also type-III and denoted as 2HDM-III where FCNC are induced at tree-level [26] which can lead to fine-tuning issues. The discussion of the different models is not crucial as the production processes discussed here have a very mild dependence on the diagrams with fermion loops. It can however become relevant when discussing the different final states. When the Higgs decays predominantly to fermions, the relative size of the $h^{0} \rightarrow b \bar{b}, h^{0} \rightarrow c \bar{c}$ and $h^{0} \rightarrow \tau^{+} \tau^{-}$branching ratios do depend on the model chosen regarding the Yukawa sector. Finally, we note that a 2HDM-I can lead to a fermiophobic Higgs boson $h^{0}$ [27] with suppressed couplings to the fermions (exactly zero at tree-level). In this case, the dominant decay mode for the lightest Higgs boson is $h^{0} \rightarrow \gamma \gamma$ or $h^{0} \rightarrow W^{+} W^{-}$, depending on its mass. No other version of the 2HDM possesses such a feature.

The most general scalar potential, renormalizable, CP-conserving, invariant under $S U(2)_{L} \otimes U(1)_{Y}$ can be written as [22]:

$$
\begin{aligned}
V\left(\Phi_{1}, \Phi_{2}\right) & =m_{1}^{2} \Phi_{1}^{\dagger} \Phi_{1}+m_{2}^{2} \Phi_{2}^{\dagger} \Phi_{2}+\left(m_{12}^{2} \Phi_{1}^{\dagger} \Phi_{2}+\text { h.c }\right)+\frac{1}{2} \lambda_{1}\left(\Phi_{1}^{\dagger} \Phi_{1}\right)^{2}+\frac{1}{2} \lambda_{2}\left(\Phi_{2}^{\dagger} \Phi_{2}\right)^{2} \\
& +\lambda_{3}\left(\Phi_{1}^{\dagger} \Phi_{1}\right)\left(\Phi_{2}^{\dagger} \Phi_{2}\right)+\lambda_{4}\left(\Phi_{1}^{\dagger} \Phi_{2}\right)\left(\Phi_{2}^{\dagger} \Phi_{1}\right)+\frac{1}{2} \lambda_{5}\left[\left(\Phi_{1}^{\dagger} \Phi_{2}\right)^{2}+\text { h.c. }\right]
\end{aligned}
$$

where $\Phi_{1}$ and $\Phi_{2}$ have weak hypercharge $Y=1$ and vacuum expectation values (VEV's) $v_{1}$ and $v_{2}$, respectively, and $\lambda_{i}$ and $m_{12}^{2}$ are real-valued parameters. Note that this potential violates the $Z_{2}$ discrete symmetry $\Phi_{1} \rightarrow \Phi_{1}, \Phi_{2} \rightarrow-\Phi_{2}$ softly by the dimension-two term $m_{12}^{2}\left(\Phi_{1}^{\dagger} \Phi_{2}\right)$, and has the same general structure as the scalar potential in the MSSM. From hermeticity of the potential, one concludes that $m_{12}^{2}$ must be real valued, so it can take both positive and negative values. 
After electroweak symmetry breaking, the $W^{ \pm}$and $Z$ gauge bosons acquire their masses. Explicitly, three of the eight degrees of freedom in the two Higgs doublets correspond to the three Goldstone bosons $\left(G^{ \pm}, G^{0}\right)$ and the remaining five become physical Higgs bosons: $h^{0}$, $H^{0}$ (CP-even), $A^{0}$ (CP-odd), and $H^{ \pm}$with masses $m_{h^{0}}, m_{H^{0}}, m_{A^{0}}$, and $m_{H^{ \pm}}$, respectively.

The potential in Eq. (11) has ten independent parameters (including $v_{1}$ and $v_{2}$ ). The parameters $m_{1}$ and $m_{2}$ are fixed by the minimization conditions. The combination $v^{2}=$ $v_{1}^{2}+v_{2}^{2}$ is fixed as usual by the electroweak breaking scale through $v^{2}=\left(2 \sqrt{2} G_{F}\right)^{-1}$. We are thus left with seven independent parameters; namely $\left(\lambda_{i}\right)_{i=1, \ldots, 5}, m_{12}$, and $\tan \beta \equiv v_{2} / v_{1}$. Equivalently, we can take instead

$$
m_{h^{0}} \quad, \quad m_{H^{0}}, m_{A^{0}}, m_{H^{ \pm}}, \tan \beta, \alpha \text { and } \mathrm{m}_{12}
$$

as the seven independent parameters. The angle $\beta$ diagonalizes both the $\mathrm{CP}$-odd and charged scalar mass matrices and $\alpha$ diagonalizes the CP-even mass matrix. One can easily relate the physical scalar masses and mixing angles from Eq. (11) to the potential parameters, $\lambda_{i}, m_{12}$ and $v_{i}$, and invert them to obtain $\lambda_{i}$ in terms of the physical scalar masses, $\tan \beta, \alpha$, and $m_{12}$ [28, 29].

\section{B. Theoretical and experimental constraints}

There are several important constraints on the 2HDM parameters imposed by experimental data. In our analysis we take them all into account when the independent parameters are varied.

First, the LEP direct search result in the lower bounds $m_{h^{0}}>114 \mathrm{GeV}$ for a SM-like Higgs and $m_{A^{0}, H^{0}, H^{ \pm}}>80-90 \mathrm{GeV}$ for supersymmetric models in the case of the neutral scalars and for more general models in the case of the charged Higgs (see [21] for details). As stated in the introduction, the bound on the lightest CP-even Higgs heavily depends on the value of $\sin (\alpha-\beta)$. In a general $2 \mathrm{HDM}$ all bounds on the Higgs masses, with the exception of the charged Higgs, can be avoided with a suitable choice of the angles and $m_{12}$.

Second, the extra contributions to the $\delta \rho$ parameter from the Higgs scalars [30] should

not exceed the current limit from precision measurements $[21]:|\delta \rho| \lesssim 10^{-3}$. Such an extra contribution to $\delta \rho$ vanishes in the limit $m_{H^{ \pm}}=m_{A^{0}}$. To ensure that $\delta \rho$ is within the allowed range, we demand either a small splitting between $m_{H^{ \pm}}$and $m_{A^{0}}$ or a combination 
of parameters that produces the same effect.

Third, the constraint from $B \rightarrow X_{s} \gamma$ branching ratio [31, 32] gives a lower bound on the charged Higgs mass, $m_{H^{ \pm}} \gtrsim 295 \mathrm{GeV}$, in 2HDM-II. These bounds do not apply to model type-I and therefore are not taken into account in the fermiophobic scenario. Recent data from $B \rightarrow \ell \nu$ can also give a constraint on charged Higgs mass especially for large values of $\tan \beta$ in 2HDM-II [33, 34].

Fourth, values of $\tan \beta$ smaller than $\approx 1$ are disallowed both by the constraints coming from $Z \rightarrow b \bar{b}$ and from $B_{q} \bar{B}_{q}$ mixing [31]].

Finally, we should take into account the theoretical constraints. Let us start by noting that all 2HDM are protected against charge and CP-breaking [35]. We consider the perturbativity constraints on the $\lambda_{i}$ as well as the vacuum stability conditions [36] that assure that the potential is bounded from below. We require that all quartic couplings of the scalar potential Eq. 1 remain perturbative by imposing $\left|\lambda_{i}\right| \leq 8 \pi$ for all $i$. For the vacuum stability conditions we use those from [36], which are given by:

$$
\begin{gathered}
\lambda_{1}>0, \quad \lambda_{2}>0, \\
\sqrt{\lambda_{1} \lambda_{2}}+\lambda_{3}+\min \left(0, \lambda_{4}-\left|\lambda_{5}\right|\right)>0 .
\end{gathered}
$$

The above perturbative constraints are slightly less constraining than the full set of unitarity constraints [37, 38] established using the high energy approximation as well as the equivalence theorem. It turns out, that requiring only perturbativity constraints on the $\lambda^{\prime} s$ could lead to scalar particles having a decay width which could exceed their mass. The problem is cured when we use the full set of perturbative unitarity conditions which are given by

$$
\left|a_{ \pm}\right|,\left|b_{ \pm}\right|,\left|c_{ \pm}\right|,\left|d_{ \pm}\right|,\left|e_{1,2}\right|,\left|f_{ \pm}\right|,\left|g_{1,2}\right|<8 \pi
$$

with

$$
\begin{aligned}
& a_{ \pm}=\frac{3}{2}\left\{\left(\lambda_{1}+\lambda_{2}\right) \pm \sqrt{\left(\lambda_{1}-\lambda_{2}\right)^{2}+\frac{4}{9}\left(2 \lambda_{3}+\lambda_{4}\right)^{2}}\right\}, \\
& b_{ \pm}=\frac{1}{2}\left\{\left(\lambda_{1}+\lambda_{2}\right) \pm \sqrt{\left(\lambda_{1}-\lambda_{2}\right)^{2}+4 \lambda_{4}^{2}}\right\}, \\
& c_{ \pm}=d_{ \pm}=\frac{1}{2}\left\{\left(\lambda_{1}+\lambda_{2}\right) \pm \sqrt{\left(\lambda_{1}-\lambda_{2}\right)^{2}+4 \lambda_{5}^{2}}\right\}, \\
& e_{1}=\left(\lambda_{3}+2 \lambda_{4}-3 \lambda_{5}\right) \quad, \quad e_{2}=\left(\lambda_{3}-\lambda_{5}\right), \\
& f_{+}=\left(\lambda_{3}+2 \lambda_{4}+3 \lambda_{5}\right) \quad, \quad f_{-}=\left(\lambda_{3}+\lambda_{5}\right), \\
& g_{1}=g_{2}=\left(\lambda_{3}+\lambda_{4}\right) .
\end{aligned}
$$


These are very restrictive constraints on the allowed range of the parameter space. All values presented in the plots are consistent with all theoretical and experimental bounds described in this section.

III. $\gamma \gamma \rightarrow S_{i} S_{j}, S_{i, j}=h^{0}, H^{0}, A^{0}$ IN THE 2HDM

\section{A. About the one-loop calculation}

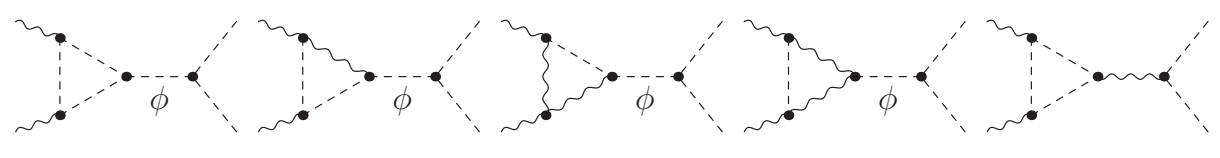

$\left(v_{1}\right)$

$\left(v_{2}\right)$

$\left(v_{3}\right)$

$\left(v_{4}\right)$

$\left(v_{5}\right)$
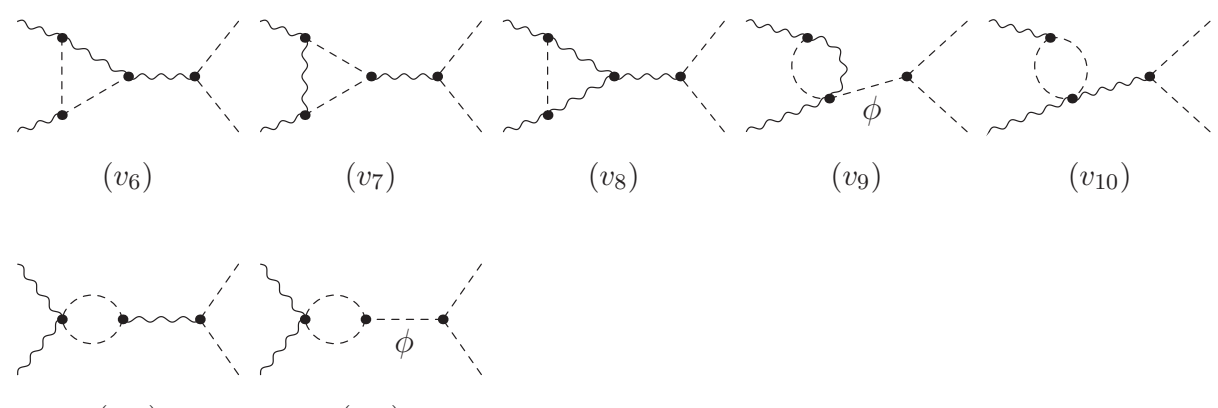

$\left(v_{11}\right) \quad\left(v_{12}\right)$

FIG. 1: Generic charged Higgs and gauge bosons vertex like Feynman diagrams for neutral Higgs production $\gamma \gamma \rightarrow S_{i} S_{j}$ in 2HDM. In the figures $\phi=h^{0}$ or $H^{0}$.

All processes $\gamma \gamma \rightarrow S_{i} S_{j}, S_{i, j}=h^{0}, H^{0}, A^{0}$ with neutral Higgs in the final state are forbidden at tree-level and are mediated at one-loop level by vertex diagrams as well as by box diagrams. All those processes are sensitive to virtual gauge bosons, fermions and charged Higgs particles. We display in Fig. 1 and in Fig. 2 the generic Feynman diagrams with charged scalar particles exchange that contribute to $\gamma \gamma \rightarrow S_{i} S_{j}$ processes. Note that in Fig. 1 and in Fig. 2 we do not show the SM contribution with fermions and gauge bosons exchange. We have checked that they are always negligible when compared to diagrams with scalar exchange. The later comprise one-loop photon-photon fusion diagrams resulting in $\phi=h^{0}$ or $H^{0}$ intermediate states, followed by the decay $h^{0}, H^{0} \rightarrow S_{i} S_{j}$ with $S_{i, j}=h^{0}, A^{0}$ 


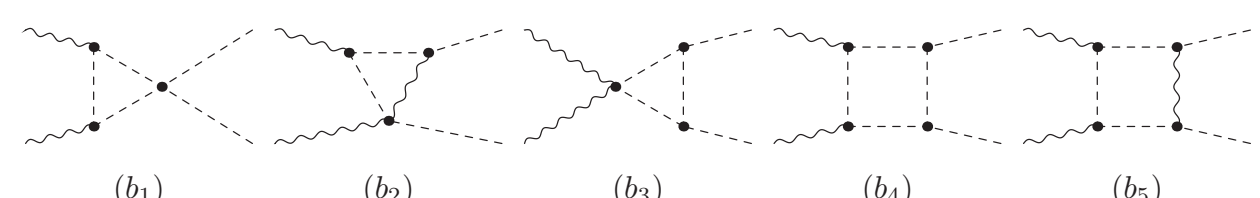

$\left(b_{1}\right)$

$\left(b_{2}\right)$

$\left(b_{3}\right)$

$\left(b_{4}\right)$

$\left(b_{5}\right)$

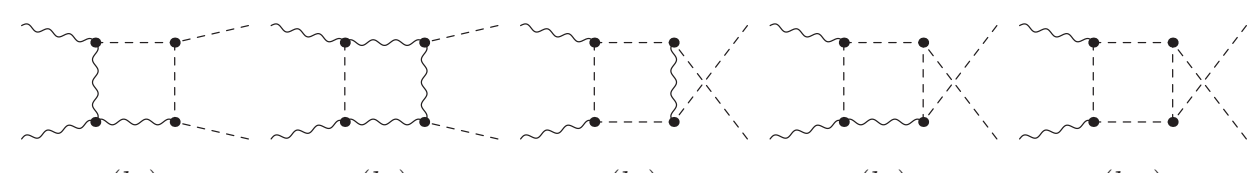

$\left(b_{6}\right)$

$\left(b_{7}\right)$

$\left(b_{8}\right)$

$\left(b_{9}\right)$

$\left(b_{10}\right)$

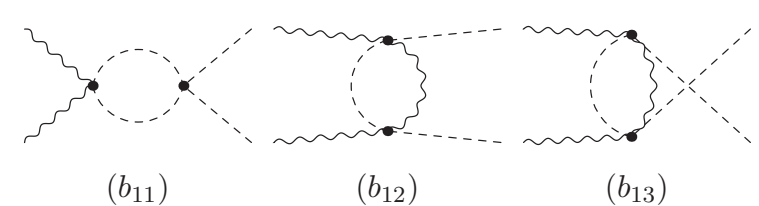

$\left(b_{11}\right)$

$\left(b_{12}\right)$

$\left(b_{13}\right)$

FIG. 2: Generic charged Higgs and gauge bosons box like Feynman diagrams to neutral Higgs production $\gamma \gamma \rightarrow S_{i} S_{j}$ in $2 \mathrm{HDM}$.

as shown in Fig. 1, $v_{1 \rightarrow 4}, v_{9}$ and $v_{12}$. This kind of topology is sensitive to the triple Higgs couplings $h^{0} S_{i} S_{j}, H^{0} S_{i} S_{j}, h^{0} H^{+} H^{-}$and $H^{0} H^{+} H^{-}$. For the $\gamma \gamma \rightarrow h^{0} A^{0}$ process, we have diagrams like $v_{1 \rightarrow 4}, v_{9}$ and $v_{12}$ but with $\phi=A^{0}$ and also the contribution from the vertices with s-channel exchange of a $Z$ boson $v_{5 \rightarrow 8}, v_{10}$ and $v_{11}$. Note that for topologies like $v_{1 \rightarrow 4}, v_{9}$ and $v_{12}$ in Fig. 1 we have included the total width of the scalar particle $\phi$ in the calculation of the corresponding amplitude.

For the production mode $\gamma \gamma \rightarrow h^{0} h^{0}$, the box contributions with virtual charged Higgs exchange is sensitive to the triple Higgs coupling $h^{0} H^{+} H^{-}$. On the contrary, in the case of the $\gamma \gamma \rightarrow A^{0} A^{0}$ process and again due to the CP nature of the pseudo-scalar Higgs boson $A^{0}$, it turns out that the box diagrams for $\gamma \gamma \rightarrow A^{0} A^{0}$ are rather sensitive to the $A^{0} H^{+} G^{-}$ coupling which does not have neither a $m_{12}$ nor a $\tan \beta$ dependence. As one can see from Fig. 2 (diagrams $\left(b_{1}\right)$ and $\left.\left(b_{11}\right)\right)$, there are other topologies that contribute to $\gamma \gamma \rightarrow h^{0} h^{0}$ and $\gamma \gamma \rightarrow A^{0} A^{0}$ and which are sensitive to quartic couplings of the Higgs boson such as $h^{0} h^{0} H^{+} H^{-}$and $A^{0} A^{0} H^{+} H^{-}$.

As stated before, we are mainly concerned with the production modes $\gamma \gamma \rightarrow h^{0} h^{0}$ and $\gamma \gamma \rightarrow A^{0} A^{0}$. We will briefly comment on the $h^{0} A^{0}, H^{0} A^{0}, h^{0} H^{0}$ and $H^{0} H^{0}$ production pro- 
cesses. The one-loop amplitudes were generated and calculated with the packages FeynArts [39] and FormCalc [40]. The scalar integrals were evaluated with LoopTools [41]. The numerical evaluations of the integration over $2 \rightarrow 2$ phase space is done by the help of CUBA library [42]. A cut of approximately $6^{\circ}$ relative to the beam axis was set on the scattering angle in the forward and backward directions.

\section{B. Triple Higgs couplings}

The above processes are sensitive to triple and quartic Higgs couplings. Below, we list the relevant pure scalar couplings needed for our processes $\gamma \gamma \rightarrow h^{0} h^{0}, A^{0} A^{0}, h^{0} A^{0}$. In the $\mathrm{SM}$ and in the general 2HDM these triple and quartic scalar couplings are given at tree-level by

$$
\begin{aligned}
& \lambda_{h^{0} h^{0} h^{0}}^{S M}=\frac{-3 g m_{h^{0}}^{2}}{2 m_{W}} \quad, \quad \lambda_{h^{0} h^{0} h^{0} h^{0}}^{S M}=\frac{-3 g^{2} m_{h^{0}}^{2}}{4 m_{W}^{2}} \\
& \lambda_{h^{0} h^{0} h^{0}}^{2 H D M}=\frac{-3 g}{m_{W} s_{2 \beta}^{2}}\left[\left(c_{\beta} c_{\alpha}^{3}-s_{\beta} s_{\alpha}^{3}\right) s_{2 \beta} m_{h^{0}}^{2}+c_{\beta-\alpha}^{2} c_{\beta+\alpha} m_{12}^{2}\right] \\
& \lambda_{H^{0} H^{0} H^{0}}^{2 H D M}=\frac{-3 g}{m_{W} s_{2 \beta}^{2}}\left[\left(c_{\beta} c_{\alpha}^{3}-s_{\beta} s_{\alpha}^{3}\right) s_{2 \beta} m_{H^{0}}^{2}+s_{\beta-\alpha}^{2} s_{\beta+\alpha} m_{12}^{2}\right] \\
& \lambda_{H^{0} h^{0} h^{0}}^{2 H D}=-\frac{1}{2} \frac{g c_{\beta-\alpha}}{s_{W} s_{2 \beta}^{2}}\left[\left(2 m_{h^{0}}^{2}+m_{H^{0}}^{2}\right) s_{2 \alpha} s_{2 \beta}+\left(3 s_{2 \alpha}-s_{2 \beta}\right) m_{12}^{2}\right] \\
& \lambda_{H^{0} H^{0} h^{0}}^{2 H D M}=\frac{1}{2} \frac{g s_{\beta-\alpha}}{m_{W} s_{2 \beta}^{2}}\left[\left(m_{h^{0}}^{2}+2 m_{H^{0}}^{2}\right) s_{2 \alpha} s_{2 \beta}+\left(3 s_{2 \alpha}+s_{2 \beta}\right) m_{12}^{2}\right] \\
& \lambda_{A^{0} A^{0} h^{0}}^{2 H D M}=\frac{-g}{m_{W} s_{2 \beta}^{2}}\left[\left(c_{\alpha} c_{\beta}^{3}-s_{\alpha} s_{\beta}^{3}\right) s_{2 \beta} m_{h^{0}}^{2}+c_{\beta+\alpha} m_{12}^{2}+s_{2 \beta}^{2} s_{\beta-\alpha} m_{A^{0}}^{2}\right] \\
& \lambda_{A^{0} A^{0} H^{0}}^{2 H D M}=\frac{-g}{m_{W} s_{2 \beta}^{2}}\left[\left(s_{\alpha} c_{\beta}^{3}+c_{\alpha} s_{\beta}^{3}\right) s_{2 \beta} m_{H^{0}}^{2}+s_{\beta+\alpha} m_{12}^{2}+s_{2 \beta}^{2} c_{\beta-\alpha} m_{A^{0}}^{2}\right] \\
& \lambda_{A^{0} G^{0} h^{0}}^{2 H D M}=\frac{1}{2} \frac{g c_{\beta-\alpha}}{m_{W}}\left(m_{A^{0}}^{2}-m_{h^{0}}^{2}\right), \lambda_{A^{0} G^{0} H^{0}}^{2 H}=-\frac{1}{2} \frac{g s_{\beta-\alpha}}{m_{W}}\left(m_{A^{0}}^{2}-m_{H^{0}}^{2}\right) \\
& \lambda_{H^{ \pm} H^{+} h^{0}}^{2 H D M}=\frac{g}{m_{W} s_{2 \beta}}\left[\left(s_{\alpha} s_{\beta}^{3}-c_{\alpha} c_{\beta}^{3}\right) s_{2 \beta} m_{h^{0}}^{2}-c_{\beta+\alpha} m_{12}^{2}-s_{2 \beta}^{2} s_{\beta-\alpha} m_{H^{ \pm}}^{2}\right] \\
& \lambda_{H^{0} A^{0} H^{-} H^{+}}^{2 H D M}=-\left(\frac{g}{2 m_{W} s_{2 \beta}}\right)^{2}\left[m_{H^{0}}^{2}\left(c_{\beta-\alpha} s_{2 \beta}-2 s_{\beta+\alpha}\right)^{2}+m_{h^{0}}^{2}\left(2 c_{\beta+\alpha}-s_{2 \beta} s_{\beta-\alpha}\right)^{2}\right. \\
& \lambda_{h^{0} h^{0} H^{-} H^{+}}^{2 H D M}=-\frac{-g}{m_{W} s_{2 \beta}}\left[\left(s_{\alpha} c_{\beta}^{3}+s_{\alpha} s_{\beta}^{3}\right) s_{2 \beta} m_{H^{0}}^{2}+s_{\beta+\alpha} m_{12}^{2}+s_{2 \beta}^{2} c_{\beta-\alpha} m_{H^{ \pm}}^{2}\right] \\
&-\left(\frac{g}{2 m_{W} s_{2 \beta}}\right)^{2}\left[2 m_{H^{ \pm}}^{2} s_{2 \beta}^{2} s_{\beta-\alpha}^{2}+m_{h^{0}}^{2}\left(2 c_{\alpha+\beta}+s_{2 \alpha} s_{\beta-\alpha}\right)\left(2 c_{\alpha+\beta}-s_{2 \beta} s_{\beta-\alpha}\right)\right. \\
&\left.\left.\lambda_{\beta-\alpha} s_{2 \beta}-2 s_{\alpha+\beta}\right) m_{H^{0}}^{2}+m_{12}^{2}\left(c_{\alpha+\beta}^{2}+c_{2 \beta}^{2} c_{\beta-\alpha}^{2}\right)\right]
\end{aligned}
$$




$$
\left.+2 m_{12}^{2} c_{2 \beta}^{2}\right]
$$

where $g=e / \sin \theta_{W}$ is the $S U(2)_{L}$ gauge coupling constant. Here we use the short-hand notations $s_{\theta}$ and $c_{\theta}$ to denote, respectively, $\sin \theta$ and $\cos \theta$ where $\theta$ stands for $\alpha$ or $\beta$. All these triple Higgs couplings have a strong dependence on the physical masses $m_{\phi}(\phi=$ $\left.h^{0}, H^{0}, H^{ \pm}, A^{0}\right)$, on the mixing angles $\alpha$ and $\beta$ and finally on the $m_{12}$ parameter which parameterizes the soft breaking of the $Z_{2}$ symmetry.
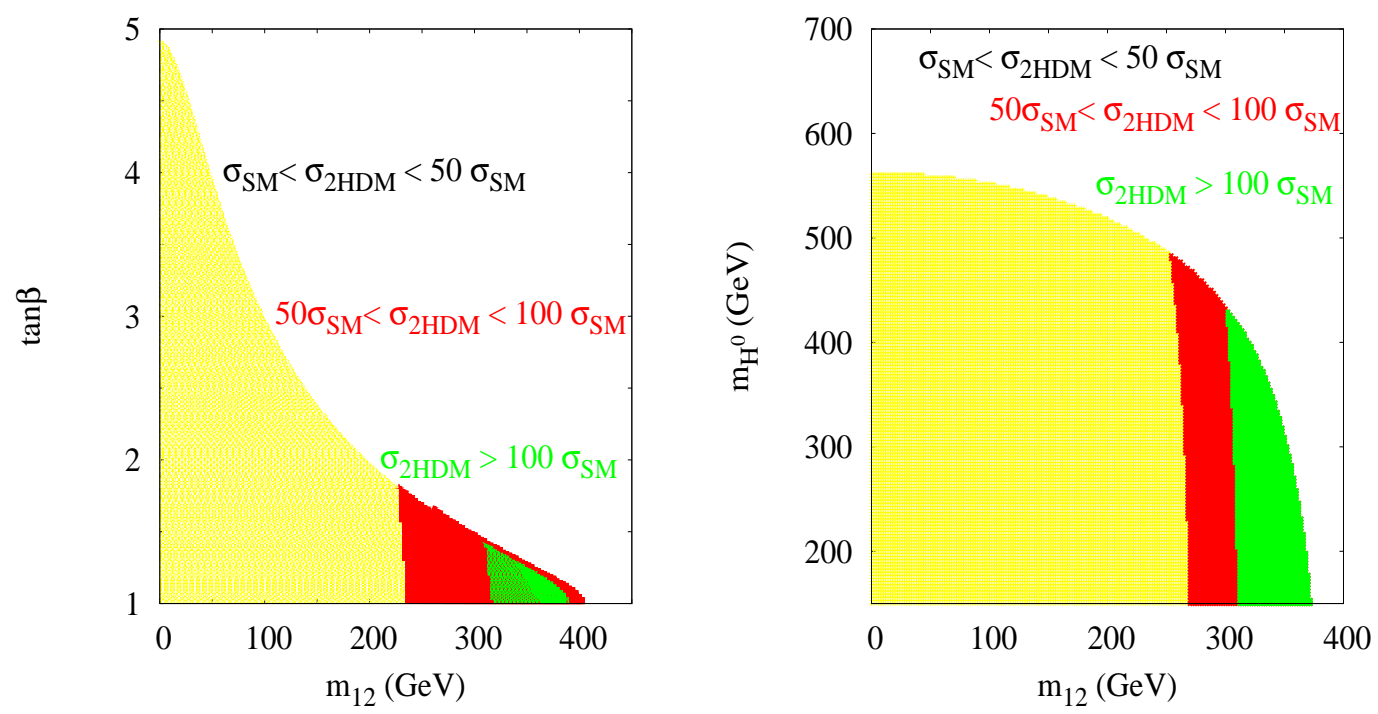

FIG. 3: The allowed regions for $\sigma\left(\gamma \gamma \rightarrow h^{0} h^{0}\right)$ in two Higgs doublet model. We have chosen $m_{h^{0}}=115 \mathrm{GeV}, m_{A^{0}}=270 \mathrm{GeV}$ and $m_{H^{ \pm}}=350 \mathrm{GeV}$. On the left panel, $m_{H^{0}}=2 m_{h^{0}}$, $E_{\gamma \gamma}=500 \mathrm{GeV},-1 \leq \sin \alpha \leq 1$ and $1 \lesssim \tan \beta \lesssim 10$. On the right panel, $\tan \beta=1, \sin \alpha=-0.9$ and $E_{\gamma \gamma}=800 \mathrm{GeV}$

\section{NUMERICAL RESULTS}

\section{A. The general $2 \mathrm{HDM}$}

Before discussing our numerical results, it is worth pointing out that the following results are valid for all Yukawa type of couplings that do not generate FCNC at tree-level, as long as $\tan \beta$ remains small ( $\tan \beta \lesssim 7$ in the regions probed), as imposed by unitarity constraints. Moreover, as we will see later, the 2HDM contribution is dominated by scalar loops rather 
than by fermion loops and the former are Yukawa model independent. Since data can easily accommodate light $h^{0}$ and $A^{0}$ scalars [20] in the 2HDM, we will concentrate hereafter on the $h^{0} h^{0}, A^{0} A^{0}$ and $h^{0} A^{0}$ production modes. In our numerical analysis, we will use: $m_{t}=171$ $\mathrm{GeV}, m_{b}=4.7 \mathrm{GeV}, m_{Z}=91.187 \mathrm{GeV}, m_{W}=80.45 \mathrm{GeV}$, the Weinberg angle $s_{W}$ is defined in the on-shell scheme as $s_{W}^{2}=1-m_{W}^{2} / m_{Z}^{2}$. For the fine structure constant we use $\alpha=1 / 137.035989$.

We first note that we have reproduced the SM result for $\gamma \gamma \rightarrow H^{0} H^{0}$ and found perfect agreement with [12, 13]. In the 2HDM case, our result agree with [14] while we have a full agreement with [15] if we take $\alpha=1 / 128$. The very detailed parton-level study [13] conclude that for a $350 \mathrm{GeV}$ center of mass energy photon collider and a Higgs mass of $120 \mathrm{GeV}$, an integrated $\gamma \gamma$ luminosity of $450 \mathrm{fb}^{-1}$ would be needed to exclude a zero trilinear Higgs boson self-coupling at the $5 \sigma$ level, considering only the statistical uncertainty. If one assumes the luminosity based on the TESLA design report [43] we conclude that this is an attainable luminosity in approximately one year and certainly in less than two years. Therefore, we have decided to perform a comprehensive scan of the parameter space of the 2HDM looking for regions where the $2 \mathrm{HDM}$ dominate over the SM, that is, $\sigma_{2 H D M}\left(\gamma \gamma \rightarrow h^{0} h^{0}\right)>\sigma_{S M}(\gamma \gamma \rightarrow$ $h^{0} h^{0}$ ), together with perturbativity, unitarity and vacuum stability constraints on $\lambda_{i}$. The results of this scan are shown in Fig. 3. In this scan we choose the charged Higgs mass to be $350 \mathrm{GeV}$, in order to fulfill the $b \rightarrow s \gamma$ constraint in all Yukawa type models. From the left scan, it comes out a correlation between $\tan \beta$ and $m_{12}$. In order to have $m_{12}$ large, unitarity constraints require $\tan \beta$ to be rather small. It is clear that in order to have a $2 \mathrm{HDM}$ cross section for $\gamma \gamma \rightarrow h^{0} h^{0}$ much larger than the corresponding SM one, a large $m_{12}$ is needed together with a small value for $\tan \beta \approx 1,1.5$. With the set of parameters fixed, unitarity constraints forces $\tan \beta \lesssim 5$ in the scanned region. The left scan shows that as $\tan \beta$ grows larger and larger, only smaller values of $m_{12}$ are allowed. In the right panel of Fig. 3 one can see that a quite large range of $m_{H}$ is allowed by perturbativity, unitarity and vacuum stability constrains. For large $m_{12} \gtrsim 260 \mathrm{GeV}$ and for all values of $m_{H} \lesssim 500 \mathrm{GeV}$, the 2HDM cross section for $\gamma \gamma \rightarrow h^{0} h^{0}$ can be 50 times larger than the corresponding SM cross section. From the scans presented, we conclude that for a large $m_{12}$ and even if $\tan \beta \approx O(1)$ we have a significant slice of the parameter space where $\sigma_{2 H D M}\left(\gamma \gamma \rightarrow h^{0} h^{0}\right)$ can be much larger than the corresponding SM cross section while complying with all constraints both experimental and theoretical. 

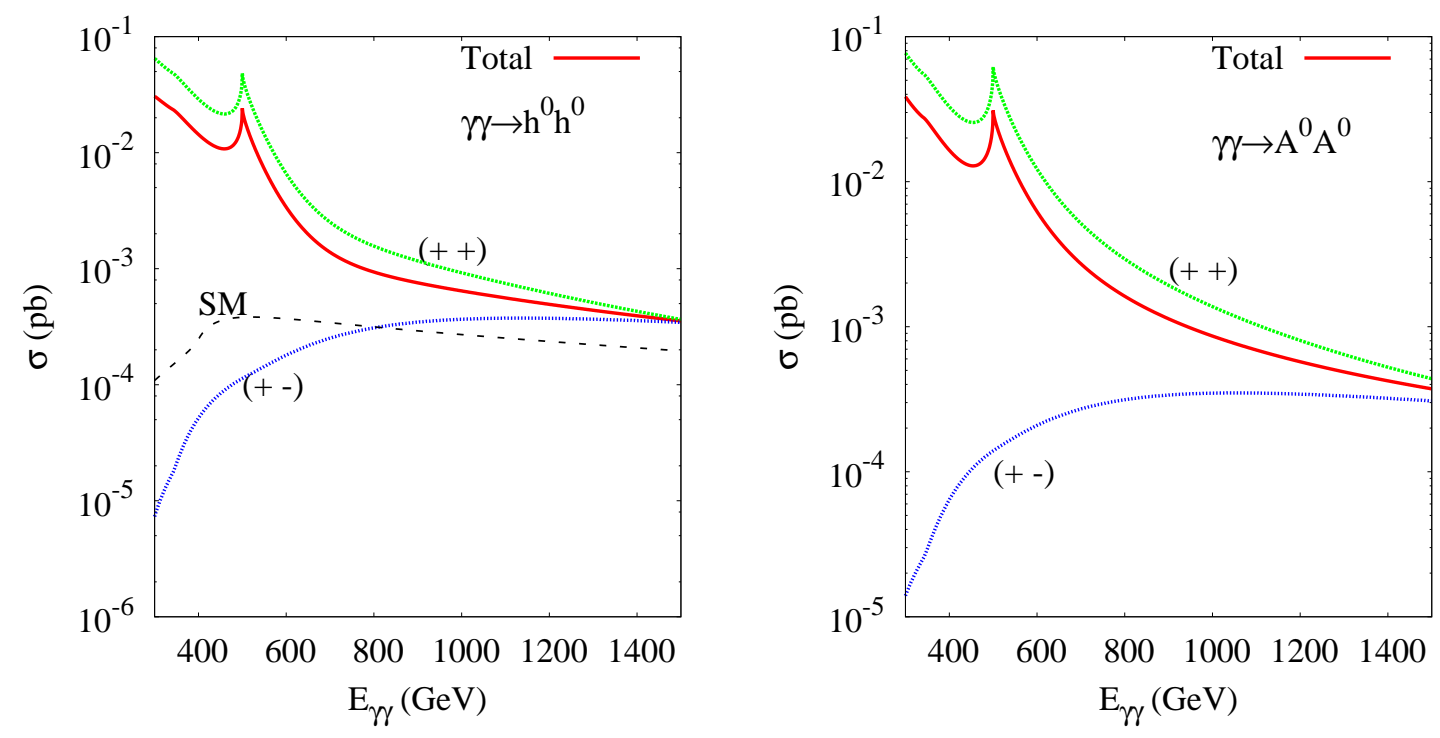

FIG. 4: SM and 2HDM total cross sections $\sigma\left(\gamma \gamma \rightarrow h^{0} h^{0}\right)$ (left) and $\sigma\left(\gamma \gamma \rightarrow A^{0} A^{0}\right)$ (right) as a function of the two photon center of mass energy for unpolarized beams. Also shown is the total cross section in two other situations: both beams with right polarization and the two beams with opposite polarization. The parameters are chosen to be $m_{h^{0}}, m_{H^{0}}, m_{A^{0}}, m_{12}=115,130,100,400$ $\mathrm{GeV}, \sin \alpha=0.6, \tan \beta=1$ and $m_{H^{ \pm}}=250 \mathrm{GeV}$.

In Fig. 4 (left) we show the polarized and unpolarized cross section for $\gamma \gamma \rightarrow h^{0} h^{0}$ both in the SM and in the 2HDM. The cross section is amplified by the threshold effect when $E_{\gamma \gamma} \approx 2 m_{H \pm}=500 \mathrm{GeV}$, corresponding to the opening of the charged Higgs pair channel, $\gamma \gamma \rightarrow H^{+} H^{-}$. Near this threshold region, the cross section of the 2HDM is more than two orders of magnitude larger than the SM one. Note that the s-channel vertex contribution is suppressed for large values of the center of mass energy due to the s-channel propagator. At high energies, box diagrams will dominates over the s-channel vertex. Let us take $h^{0} h^{0}$ production as an example. The value of the couplings for this set of parameters is

$$
\begin{array}{lc}
\lambda_{h^{0} H^{+} H^{-}}^{2 H D M} \approx 3 \times \lambda_{h^{0} h^{0} h^{0}}^{S M}, & \lambda_{h^{0} A^{0} A^{0}}^{2 H D M} \approx 2.5 \times \lambda_{h^{0} h^{0} h^{0}}^{S M} \\
\lambda_{H^{0} H^{+} H^{-}}^{2 H D M} \approx 20 \times \lambda_{h^{0} h^{0} h^{0}}^{S M}, & \lambda_{H^{0} A^{0} A^{0}}^{2 H D M} \approx 17.5 \times \lambda_{h^{0} h^{0} h^{0}}^{S M} \\
\lambda_{h^{0} h^{0} h^{0}}^{2 H D} \approx 7 \times \lambda_{h^{0} h^{0} h^{0}}^{S M}, & \lambda_{H^{0} h^{0} h^{0}}^{2 H D} \approx 16.5 \times \lambda_{h^{0} h^{0} h^{0}}^{S M} \\
\lambda_{h^{0} h^{0} H^{+} H^{-}}^{2 H D \lambda_{h^{0} h^{0} h^{0} h^{0}}}, \quad, \quad \lambda_{A^{0} A^{0} H^{+} H^{-}}^{2 H D} \approx 0.5 \times \lambda_{h^{0} h^{0} h^{0} h^{0}}^{S M} .
\end{array}
$$

Now the largest contribution to the vertex comes from the $H^{0} H^{+} H^{-}$and $H^{0} h^{0} h^{0}$ couplings squared which are approximately 400 and 272 times larger than $\left(\lambda_{h^{0} h^{0} h^{0}}^{S M}\right)^{2}$. Note that in 

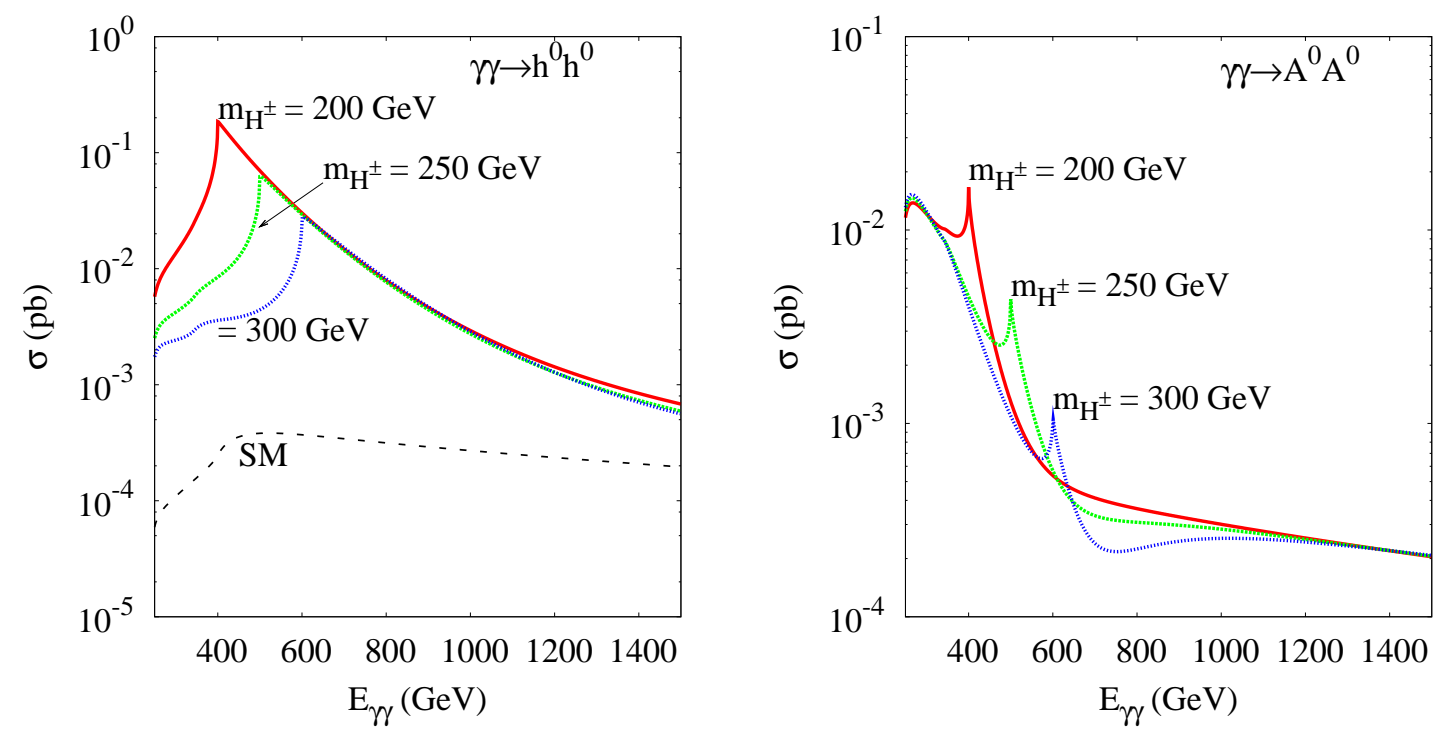

FIG. 5: The total cross section of $\sigma(\gamma \gamma \rightarrow h h)$ (left) and $\sigma\left(\gamma \gamma \rightarrow A^{0} A^{0}\right)$ (right) as a function of two photon center of mass energy in the 2HDM. With $m_{h^{0}}, m_{H^{0}}, m_{A^{0}}, m_{12}=120,200,120,300$ $\mathrm{GeV}, \sin \alpha=-0.86$ and $\tan \beta=1$ for different values of $m_{H^{ \pm}}$.

the case of $A^{0} A^{0}$ there are no boxes with virtual $H^{ \pm}$exchange because the $A^{0} H^{+} H^{-}$coupling does not exist and consequently the vertex contribution will dominate over the box contribution.

In the right panel of Fig. 4 the CP-odd Higgs boson pair production $\gamma \gamma \rightarrow A^{0} A^{0}$ is shown. The conclusions are very similar to the ones for the $h^{0} h^{0}$ final state. The total cross sections is dominated by vertex contributions for low energy. This dominance is amplified by the threshold effect when $E_{\gamma \gamma} \approx 2 m_{H \pm}$. For high center of mass energies, box contributions dominate because they have those $t$ and $u$ channel topologies which are enhanced for large center of mass energies. Finally, we show that when the initial photons are both right handed $(++)$ or left handed $(--)$ polarized, the cross sections are enhanced by more than a factor two as compared to the unpolarized case. In the opposite case, when the initial photons have opposite polarizations, $(+-)$ or $(-+)$, the cross sections are now suppressed when compared to unpolarized case.

The box contribution has either the quartic vertex $h^{0} h^{0} H^{+} H^{-}$or twice the triple vertex $h^{0} H^{+} H^{-}$which means that the cross section will have $\left(\lambda_{h^{0} H^{+} H^{-}}\right)^{4}$ dependence. When $h^{0} H^{+} H^{-}$and/or $h^{0} h^{0} H^{+} H^{-}$are large enough the boxes could dominate over the vertices. 
By tuning $\sin \alpha$ one can make $h^{0} H^{+} H^{-}$and $h^{0} h^{0} H^{+} H^{-}$large enough to allow for the boxes to dominate. This is exactly what is illustrated in the following Fig. 5, where we present the total cross section for $\gamma \gamma \rightarrow h^{0} h^{0}$ (left) and $\gamma \gamma \rightarrow A^{0} A^{0}$ (right) as a function of the two photon center of mass energy for different values of the charged Higgs mass. Once more we can see the enhancement when $E_{\gamma \gamma} \approx 2 m_{H \pm}$ for $\gamma \gamma \rightarrow h^{0} h^{0}$ and the cross section can reach 0.2 pbarn. For this specific scenario, the triple and quartic couplings are

$$
\begin{array}{lc}
\lambda_{h^{0} H^{+} H^{-}}^{2 H D M} \approx 13 \times \lambda_{h^{0} h^{0} h^{0}}^{S M}, & \lambda_{h^{0} A^{0} A^{0}}^{2 H D M} \approx 9 \times \lambda_{h^{0} h^{0} h^{0}}^{S M} \\
\lambda_{H^{0} H^{+} H^{-}}^{2 H D M} \approx 4 \times \lambda_{h^{0} h^{0} h^{0}}^{S M}, & \lambda_{H^{0} A^{0} A^{0}}^{2 H D M} \approx 2 \times \lambda_{h^{0} h^{0} h^{0}}^{S M} \\
\lambda_{h^{0} h^{0} h^{0}}^{2 H D} \approx 3 \times \lambda_{h^{0} h^{0} h^{0}}^{S M}, & \lambda_{H^{0} h^{0} h^{0}}^{2 H D M} \approx 3 \times \lambda_{h^{0} h^{0} h^{0}}^{S M} \\
\lambda_{h^{0} h^{0} H^{+} H^{-}}^{2 H D} \approx 12 \times \lambda_{h^{0} h^{0} h^{0} h^{0}}^{S M}, \quad \lambda_{A^{0} A^{0} H^{+} H^{-}}^{2 H D M} \approx 0.5 \times \lambda_{h^{0} h^{0} h^{0} h^{0}}^{S M}
\end{array}
$$

In the case of the $\gamma \gamma \rightarrow h^{0} h^{0}$ mode, the total cross section is now fully dominated by box contributions both at low and high energies. This is because the couplings $h^{0} H^{+} H^{-}$and $h^{0} h^{0} H^{+} H^{-}$which contribute to the boxes are relatively large. In the case of $\gamma \gamma \rightarrow A^{0} A^{0}$ mode, for low energy, the total cross section is dominated by vertex diagrams because the triple couplings $h^{0} H^{+} H^{-}$and $h^{0} A^{0} A^{0}$ are large. For high energies, where vertex are suppressed, the total cross section is dominated by the box contributions. Hence, at high energies, both for the $\gamma \gamma \rightarrow h^{0} h^{0}$ and for the $\gamma \gamma \rightarrow A^{0} A^{0}$ modes, the total contribution is dominated by boxes.

In Fig. 6, we illustrate the sensitivity to the charged Higgs boson contribution for different center of mass energy for the $h^{0} h^{0}$ and the $A^{0} A^{0}$ modes. In both cases, the cross sections are enhanced for light charged Higgs for the reasons explained above and are suppressed after crossing the threshold for $\gamma \gamma \rightarrow H^{+} H^{-}$production, i.e., $m_{H \pm} \gtrsim E_{\gamma \gamma} / 2$.

In Fig. 7 we show the total cross section for $\gamma \gamma \rightarrow h^{0} h^{0}$ (left) and $\gamma \gamma \rightarrow A^{0} A^{0}$ (right) as a function the heavy Higgs mass for several values of the center of mass energy. Once the center of mass energy is close to $m_{H^{0}}$, one can see in both plots the effect of the resonance of the heavy CP-even Higgs. The difference between them is only due to the vertex diagrams with an intermediate heavy Higgs that then decays to $h^{0} h^{0}$ or $A^{0} A^{0}$. In both cases, the cross sections can reach $0.1 \mathrm{pb}$ near the resonance $E_{\gamma \gamma} \approx m_{H^{0}}$.

For the $h^{0} H^{0}$ or $H^{0} H^{0}$ final states, the generic set of Feynman diagrams that contribute to those processes is almost the same. The main enhancement factor for the cross section is 

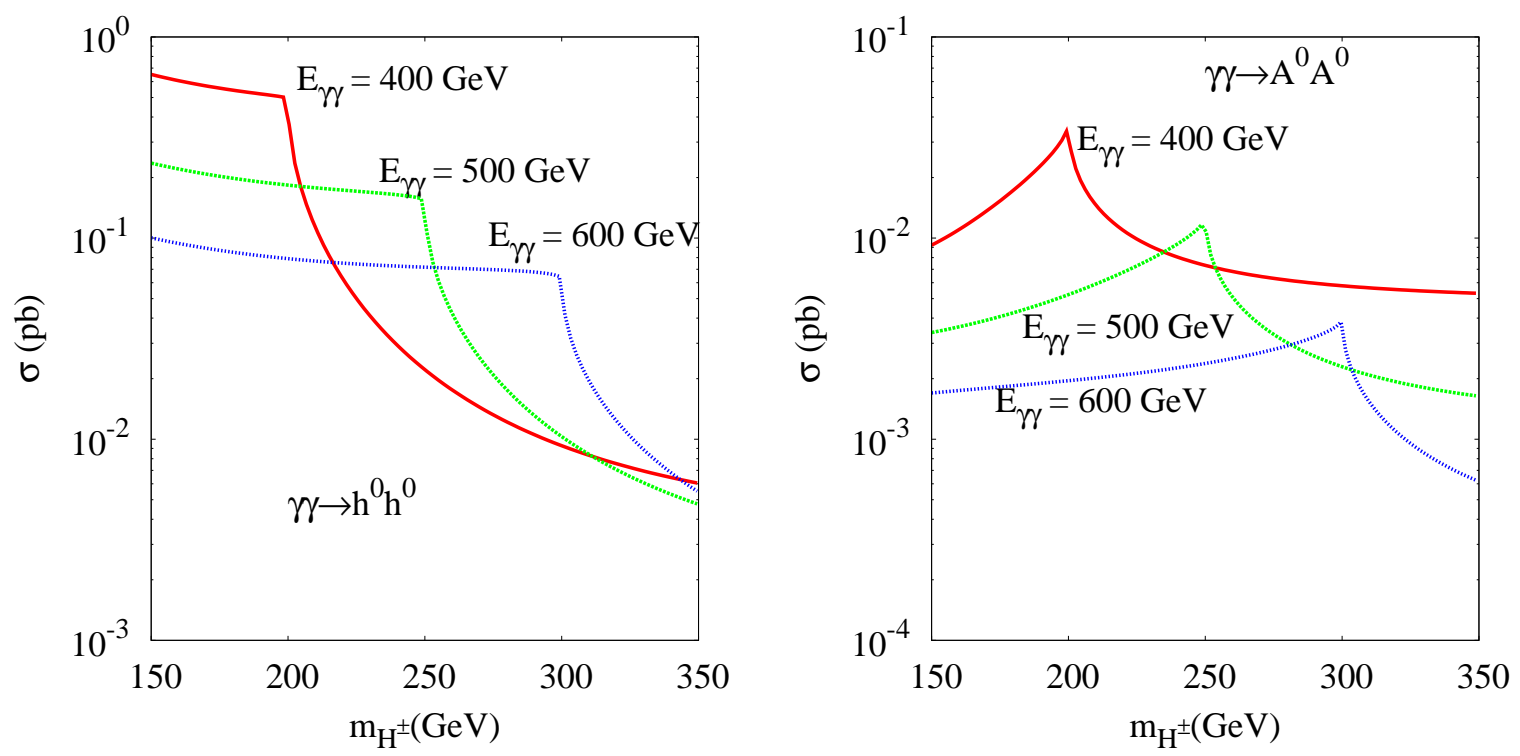

FIG. 6: The total cross section $\sigma\left(\gamma \gamma \rightarrow h^{0} h^{0}\right)$ (left) and $\sigma\left(\gamma \gamma \rightarrow A^{0} A^{0}\right)$ (right) as a function of the charged Higgs mass for different center of mass energies $E_{\gamma \gamma}$ in the 2HDM. With $m_{h^{0}}, m_{H^{0}}, m_{A^{0}}, m_{12}=120,240,270,350 \mathrm{GeV}, \sin \alpha=-0.9$ and $\tan \beta=1$.
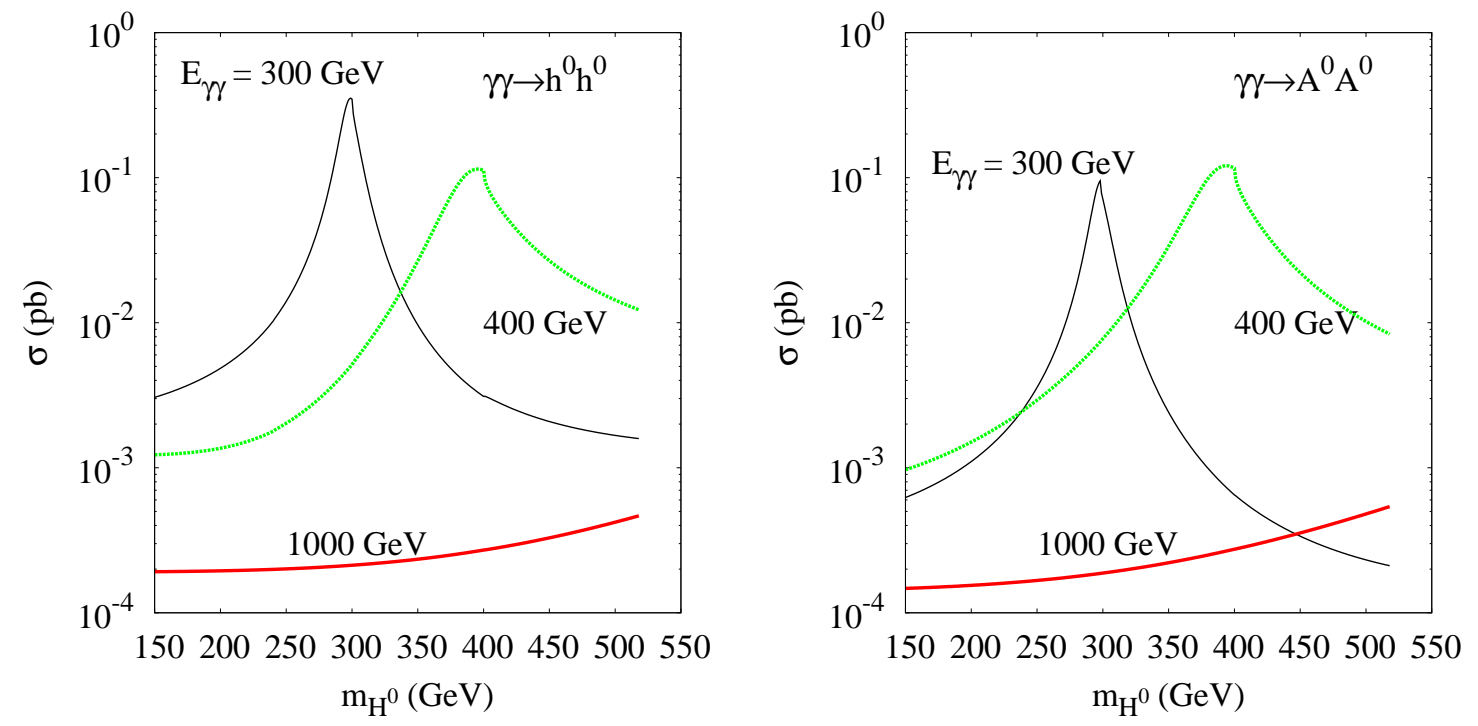

FIG. 7: The total cross section $\sigma\left(\gamma \gamma \rightarrow h^{0} h^{0}\right)$ (left) and $\sigma\left(\gamma \gamma \rightarrow A^{0} A^{0}\right)$ (right) as a function of the heavy Higgs mass $m_{H^{0}}$ in the 2HDM. With $m_{h^{0}}, m_{H^{ \pm}}, m_{A^{0}}, m_{12}=120,250,150,200 \mathrm{GeV}$, $\sin \alpha=0.9$ and $\tan \beta=1.5$ for different values of the two photon center of mass energy $E_{\gamma \gamma}$. 

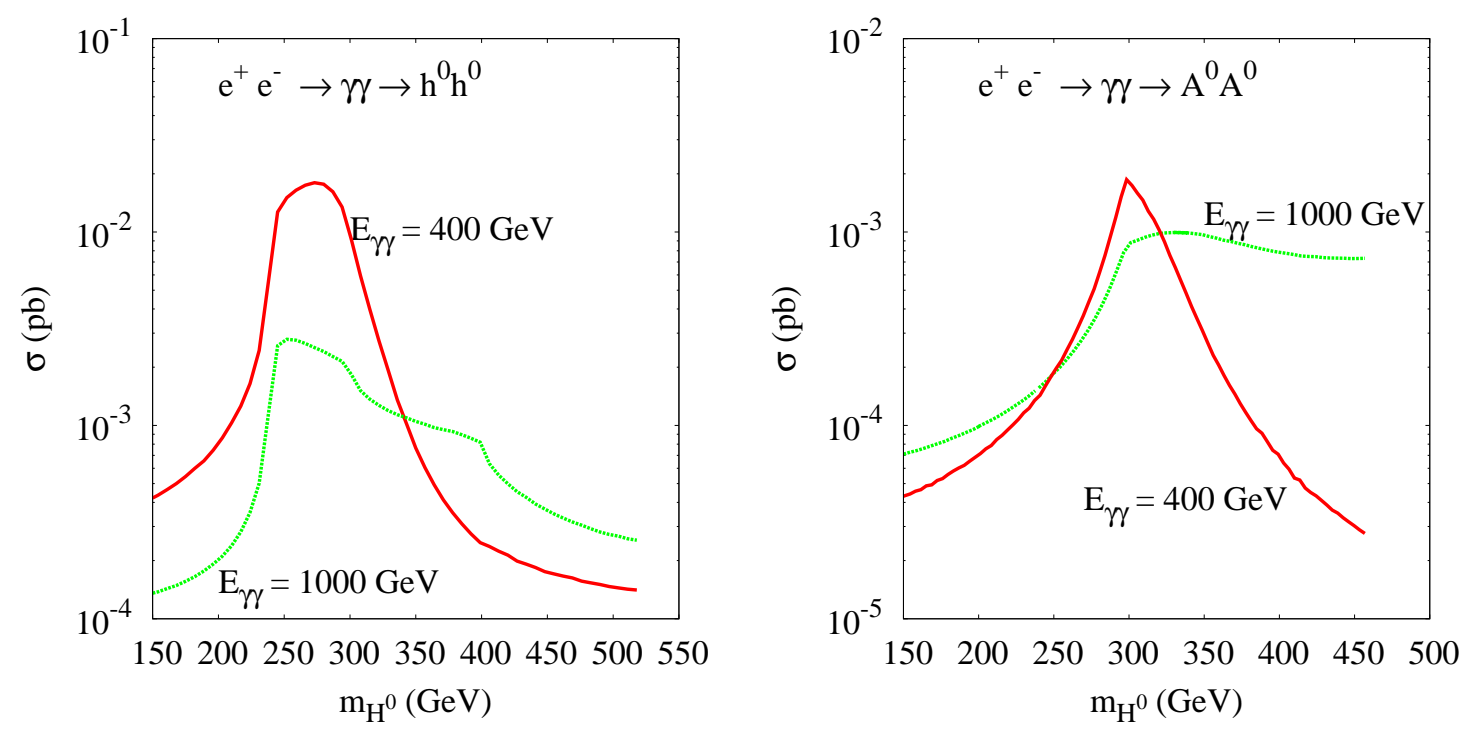

FIG. 8: The total cross section $\sigma\left(e^{+} e^{-} \rightarrow \gamma \gamma \rightarrow h^{0} h^{0}\right)$ (left) and $\sigma\left(e^{+} e^{-} \rightarrow \gamma \gamma \rightarrow A^{0} A^{0}\right)$ (right) as a function of the heavy Higgs mass $m_{H^{0}}$ in the $2 \mathrm{HDM}$ with the same parameters as in Fig. 7

the virtual charged Higgs bosons exchange, particularly relevant near the threshold region $E_{\gamma \gamma}=2 m_{H \pm}$. The only difference between those final states and the $h^{0} h^{0}$ and the $A^{0} A^{0}$ ones, is the absence of the $H^{0}$ resonant effect, since it can not decay neither to $h^{0} H^{0}$ nor to $H^{0} H^{0}$. The situation is the same as in the SM. If the CP-even $H^{0}$ has a mass of the same order as $h^{0}$ the cross section of $h^{0} H^{0}$ or $H^{0} H^{0}$ could be of the same order of magnitude and may reach $0.1 \mathrm{pb}$. If the CP-even Higgs is heavy, phase space suppression occurs and the cross sections for $h^{0} H^{0}$ and $H^{0} H^{0}$ production are smaller.

In the case of $h^{0} A^{0}$ or $H^{0} A^{0}$ final state, a quite different situation occurs. Due to the presence of the CP odd scalar $A^{0}$ in the final state, the Higgs boson $\phi$ in the s-channel vertex (Fig. 1) $v_{1 \rightarrow 4}, v_{9}$ and $v_{12}$ must also be CP-odd. Hence, the processes $\gamma \gamma \rightarrow h^{0} A^{0}, H^{0} A^{0}$ do not proceed through an intermediate CP-even Higgs. This implies that there is no closed loop of virtual exchange of charged Higgs, both in the vertex and box contributions, which again, is the main factor of enhancement of the cross section for the $h^{0} h^{0}$ and the $A^{0} A^{0}$ modes. In the boxes, we have rather a mixture of charged Higgs and charged Goldstones in the loop (gauge couplings). We have checked that the values of the cross section of $\gamma \gamma \rightarrow h^{0} A^{0}$ are much smaller than the $h^{0} h^{0}$ and $A^{0} A^{0}$ ones. We have performed a systematic scan over the 2HDM parameters with $m_{h^{0}}=m_{A^{0}}$ in the range 100 to $160 \mathrm{GeV}$, and found that the cross section of $\gamma \gamma \rightarrow h^{0} A^{0}$ does not exceed $0.04 p b$. Note that the largest value for the cross 
section, $0.04 \mathrm{pb}$, is attained near the top quark threshold region $E_{\gamma \gamma} \approx 2 m_{t}$; away from this region the cross section drops below $0.01 \mathrm{pb}$. Similarly, we found that large cross section for $\gamma \gamma \rightarrow h^{0} A^{0}$ prefer rather small values of $\tan \beta \lesssim 3,|\sin \alpha| \gtrsim 0.5$, large $m_{12}$ and also low center of mass energies $\sqrt{s} \lesssim 600 \mathrm{GeV}$.

Finally, in Fig. 8 we show the total cross section for $e^{+} e^{-} \rightarrow \gamma \gamma \rightarrow h^{0} h^{0}$ (left) and for $e^{+} e^{-} \rightarrow \gamma \gamma \rightarrow A^{0} A^{0}$ (right) as a function the heavy Higgs mass for two center of mass energies $E_{\gamma \gamma}$. The total cross section is evaluated by convoluting the photon-photon cross section with the photon-photon luminosity spectrum taken from the CompAZ library [44]. CompAZ is based on formulae for the Compton scattering and provides the photon energy spectrum for different beam energies and the average photon polarization for a given photon energy. First, let us remark that again this cross section is large enough to be measured in a significant region of the parameter space. We can still see the heavy Higgs resonance effects but somehow softened by the photon spectrum.
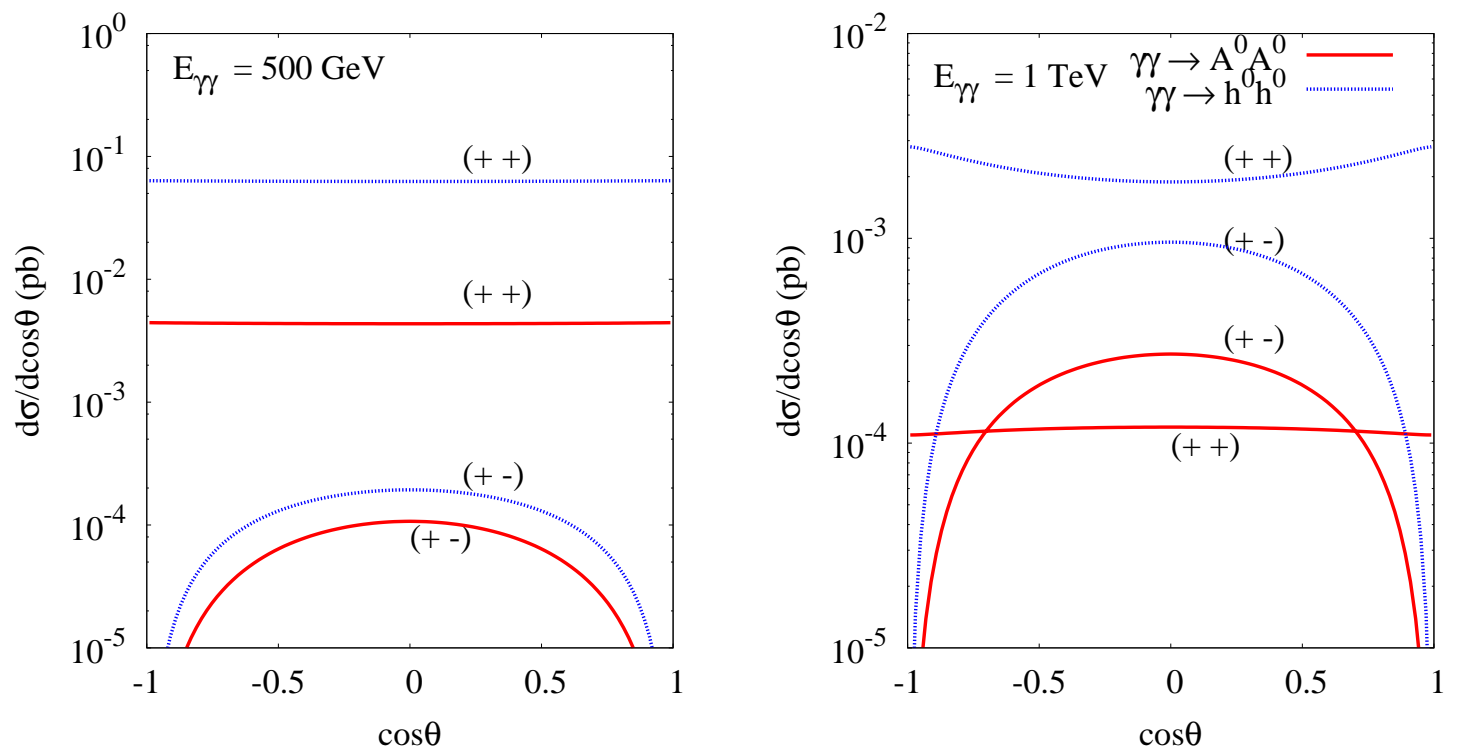

FIG. 9: The differential cross section for $\gamma \gamma \rightarrow h^{0} h^{0}$ and $\gamma \gamma \rightarrow A^{0} A^{0}$ with equally polarized photons and oppositely polarized photons for $\sqrt{s}=500 \mathrm{GeV}$ (left) and $\sqrt{s}=1 \mathrm{TeV}$ (right). The 2HDM parameters are the same as in Fig 5 and $m_{H^{ \pm}}$fixed at $250 \mathrm{GeV}$.

Let us now turn to the differential cross section for $\gamma \gamma \rightarrow h^{0} h^{0}$ and $\gamma \gamma \rightarrow A^{0} A^{0}$. In Fig. 9 we illustrate the differential cross for a center of mass energy of $\sqrt{s}=500 \mathrm{GeV}$ and $\sqrt{s}=1$ $\mathrm{TeV}$ and for identically polarized $(++),(--)$ and oppositely polarized $(+-),(-+)$ initial photons. As one can see from the left panel for $\sqrt{s}=500 \mathrm{GeV}$, for both the $h^{0} h^{0}$ and 
the $A^{0} A^{0}$ modes, the angular distribution for $(++)$ and $(--)$ polarized photons is almost flat while for the $(+-)$ and $(-+)$ modes it has a parabolic shape with a rather small cross section except for $\gamma \gamma \rightarrow h^{0} h^{0}$ where in the region of $-0.5<\cos \theta<0.5$ the differential cross section is of the order of $0.1 \mathrm{fb}$. For $\sqrt{s}=1 \mathrm{TeV}$, Fig. 9 (right panel), it is clear that the angular distribution are very similar to the ones on the left panel with the cross sections more than one order of magnitude smaller. The shape of these differential cross sections clearly tell us that we will not be able to distinguish the $\mathrm{CP}$ nature of the particle on the basis of this angular distribution.

\section{B. Fermiophobic limit}

In the SM, where just one doublet couples to all fermions, each scalar couples to the different fermions with the same coupling constant. In a general $2 \mathrm{HDM}$ it is also possible to couple just one doublet to all fermions by choosing an appropriate symmetry for both the fermions and the scalars. However, the difference between the SM and the 2HDM is that now the couplings are proportional to the rotation angles $\alpha$ and $\beta$. For instance, the lightest CP-even Higgs couples to all fermions as $\cos \alpha / \sin \beta g_{h f \bar{f}}^{S M}$. By choosing $\alpha= \pm \frac{\pi}{2}$, the lightest Higgs decouples from all fermions. Such a scenario, with the appearance of the so-called "fermiophobic" Higgs boson, arise in a variety of models [27]. The heavy CP-even scalar will acquire larger couplings to the fermions than the corresponding SM couplings. All the remaining scalars are not affected by this choice as they do not couple proportionally to $\alpha$.

In the situation where the Higgs-fermion couplings are substantially suppressed, the full decay width of the Higgs boson is shared mostly between the $W W, Z Z$ and $\gamma \gamma$ decay modes. In this limit, for masses $m_{h^{0}}<100 \mathrm{GeV}$, the Higgs boson dominantly decays to photon pairs. Experimental searches for fermiophobic Higgs bosons at the LEP collider and the Tevatron collider have yielded negative results. Mass limits have been set in a benchmark model that assumes that the couplings $h W W$ and $h Z Z$ have the same strength as in the SM and that all fermion branching ratios are exactly zero. Combination of the results obtained by the LEP collaborations [45, 46, 47, 48] using the process $e^{+} e^{-} \rightarrow h Z, h \rightarrow \gamma \gamma$ yielded the lower bound $m_{h}>109.7 \mathrm{GeV}$ at $95 \%$ C.L. [49]. In Run I, Tevatron has set lower limits on $m_{h}$

by the D0 and CDF collaborations which are respectively $78.5 \mathrm{GeV}$ [50] and $82 \mathrm{GeV} \mathrm{[51],}$ using the processes $q \bar{q}^{\prime} \rightarrow V^{*} \rightarrow h W, h Z, h \rightarrow \gamma \gamma$, with the dominant contribution coming 

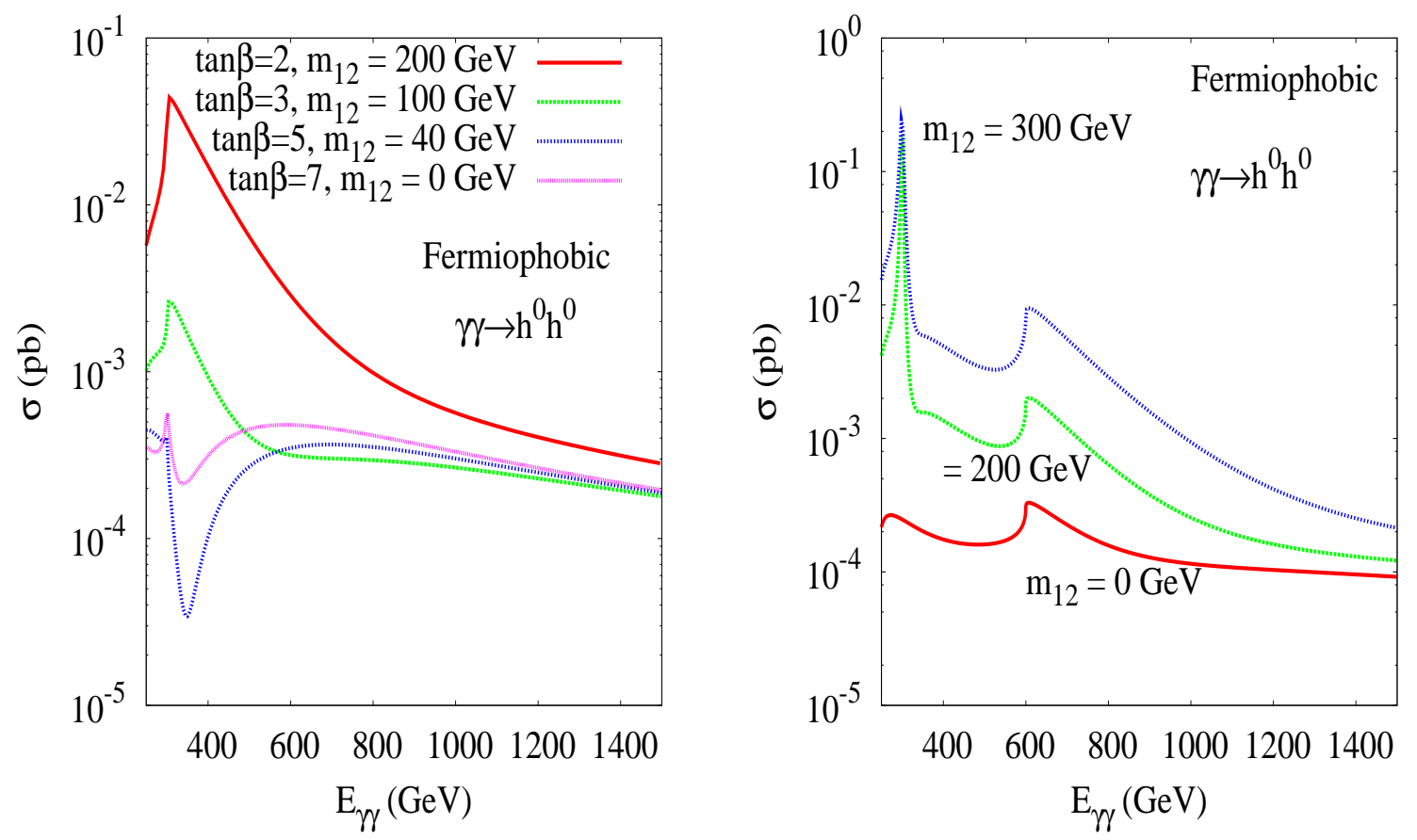

FIG. 10: The total cross section $\sigma\left(\gamma \gamma \rightarrow h^{0} h^{0}\right)$ as a function of the center of mass energy $E_{\gamma \gamma}$ in the fermiophobic limit $(\sin \alpha=1)$. In the left panel with different values of $\tan \beta$ and $m_{12}$ for $m_{h^{0}}, m_{H^{0}}, m_{A^{0}}, m_{H^{ \pm}}=100,200,100,150 \mathrm{GeV}$. In the right panel, $m_{h^{0}}=120 \mathrm{GeV}, m_{H^{0}}=$ $m_{H^{ \pm}}=m_{A^{0}}=300 \mathrm{GeV}$ and $\tan \beta=1$.

from the $W$ boson. Recently the CDF [52] and the D0 [53] collaborations have improved their bounds which are now of the order of $100 \mathrm{GeV}$, close to the bound obtained by the LEP collaborations. It should be noted that all experimental mass bounds assume, in the fermiophobic limit, $\tan \beta \sim 0$ in the tree-level couplings to the gauge bosons.

It is clear that in the fermiophobic limit the coupling $H^{0} h^{0} h^{0}$ (Eq. (14)) is directly proportional to $m_{12}$, while the other couplings depend both on $m_{12}, \tan \beta$ as well as on $m_{H^{ \pm}}$. We show in Fig. 10, the total cross section for $\gamma \gamma \rightarrow h^{0} h^{0}$ as a function of the two photon center of mass energy in the fermiophobic limit. The parameters $m_{12}$ and $\tan \beta$ are varied within the allowed range. It is clear that the cross section is enhanced for large $m_{12}$ and can reach $0.1 \mathrm{pb}$ for large $m_{12}=200 \mathrm{GeV}$ (left) and $m_{12}=300 \mathrm{GeV}$ (right). The observed kinks are the top threshold at $E_{\gamma \gamma}=2 m_{t}$ and the charged Higgs threshold $E_{\gamma \gamma}=2 m_{H \pm}$. 


\section{Decoupling limit}

A study of $2 \mathrm{HDM}$ in the decoupling limit reveals the case where all scalar masses except one formally become large and the effective theory is just the SM with one doublet $-m_{h^{0}}<<$ $m_{\Phi}$ where $m_{\Phi}=m_{H^{0}, A^{0}, H^{ \pm}}$(see [28] for an overview). In this case, the CP-even $h^{0}$ is the lightest scalar particle while the other Higgs particles $H^{0}, A^{0}$ and $H^{ \pm}$are extremely heavy. In 2HDM, the decoupling limit can be achieved by taking the limit $\alpha \rightarrow \beta-\pi / 2$. This means that the coupling of the $h^{0}$ to the gauge bosons, fermions and light Higgs, $h^{0}$ are the same as for the Standard Model $h^{S M}$ Higgs. Also, in the decoupling limit, the triple Higgs coupling $\lambda_{h^{0} h^{0} H^{0}}^{(0)}$ vanishes at tree-level, so that the heavy Higgs cannot contribute to the process $\gamma \gamma \rightarrow h^{0} h^{0}$ and the result is independent of the mass $m_{H^{0}}$. In the decoupling limit, the tree-level trilinear Higgs couplings take the form

$$
\begin{aligned}
\lambda_{h^{0} h^{0} h^{0}}^{2 H D M} & \approx \lambda_{h^{0} h^{0} h^{0}}^{S M}, \\
\lambda_{h^{0} h^{0} H^{0}}^{2 H D M} & \approx 0 \\
\lambda_{h^{0} H^{+} H^{-}}^{2 H D M} & \approx-\frac{g}{2 m_{W}}\left[m_{h^{0}}^{2}+2 m_{H^{ \pm}}^{2}+m_{12}^{2}\right], \\
\lambda_{h^{0} h^{0} H^{+} H^{-}}^{2 H D M} & \approx \frac{g}{2 m_{W}} \lambda_{h^{0} H^{+} H^{-}}^{2 H D M} .
\end{aligned}
$$

It is clear that these couplings are independent of $\tan \beta$ as well. As one can see from the analytical expression of $\lambda_{h^{0} H^{+} H^{-}}^{2 H D M}$ and $\lambda_{h^{0} h^{0} H^{+} H^{-}}^{2 H D M}$, the charged Higgs and $m_{12}^{2}$ add constructively for $m_{12}^{2}>0$ and destructively for $m_{12}^{2}<0$. At tree level, the enhancement of the cross section essentially depends on the size of the $h^{0} H^{+} H^{-}$and $H^{0} H^{-} H^{+}$couplings. By taking these couplings as large as possible under the referred theoretical and experimental constraints, we obtain the best possible enhancement for the cross section $\sigma\left(\gamma \gamma \rightarrow h^{0} h^{0}\right)$ in the $2 \mathrm{HDM}$ for each $m_{\Phi}\left(m_{\Phi}=m_{H^{0}}=m_{A^{0}}=m_{H^{ \pm}}\right)$. However, it is well known that those couplings of the CP-even $h^{0}$ which mimic the SM couplings get significant radiative corrections in the decoupling limit to which we refer to as non-decoupling effects. Several studies have been carried out looking for non-decoupling effects in Higgs boson decays and Higgs self-interactions. Large loop effects in $h^{0} \rightarrow \gamma \gamma, h^{0} \rightarrow \gamma Z$ and $h^{0} \rightarrow b \bar{b}$ have been pointed out for the 2HDM [54, 55] and may provide indirect information on the Higgs masses and

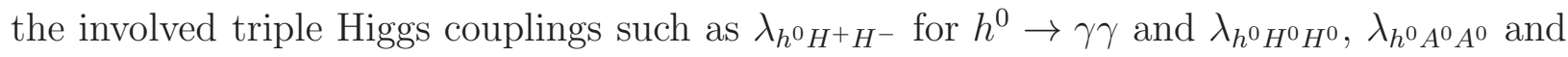
$\lambda_{h^{0} h^{0} h^{0}}$ for $h^{0} \rightarrow b \bar{b}$.

The non-decoupling contributions to the triple Higgs self-coupling $\lambda_{h^{0} h^{0} h^{0}}$ have been in- 
vestigated in the 2HDM in Ref. [56], using the Feynman diagrammatic method. It has been demonstrated that the one-loop leading contributions originated from the heavy Higgs boson loops and the top quark loops to the effective $h^{0} h^{0} h^{0}$ coupling can be written as [56]

$$
\begin{aligned}
\lambda_{h^{0} h^{0} h^{0}}^{e f f} & =\frac{3 m_{h^{0}}^{2}}{v}\left\{1+\frac{m_{H^{0}}^{4}}{12 \pi^{2} m_{h^{0}}^{2} v^{2}}\left(1+\frac{M^{2}}{m_{H^{0}}^{2}}\right)^{3}+\frac{m_{A^{0}}^{4}}{12 \pi^{2} m_{h^{0}}^{2} v^{2}}\left(1+\frac{M^{2}}{m_{A^{0}}^{2}}\right)^{3}\right. \\
& \left.+\frac{m_{H^{ \pm}}^{4}}{6 \pi^{2} m_{h^{0}}^{2} v^{2}}\left(1+\frac{M^{2}}{m_{H^{ \pm}}^{2}}\right)^{3}-\frac{N_{c} m_{t}^{4}}{3 \pi^{2} m_{h^{0}}^{2} v^{2}}+\mathcal{O}\left(\frac{p_{i}^{2} m_{\Phi}^{2}}{m_{h^{0}}^{2} v^{2}}, \frac{m_{\Phi}^{2}}{v^{2}}, \frac{p_{i}^{2} m_{t}^{2}}{m_{h^{0}}^{2} v^{2}}, \frac{m_{t}^{2}}{v^{2}}\right)\right\},
\end{aligned}
$$

where $M^{2}=m_{12}^{2} /(\sin \beta \cos \beta), m_{\Phi}$ and $p_{i}$ represent the mass of $H^{0}, A^{0}$ or $H^{ \pm}$and the momenta of external Higgs lines, respectively, $N_{c}$ denotes the number of colors, and $m_{t}$ is the mass of top quark. We note that in Eq. (27) $m_{h^{0}}$ is the renormalized physical mass of the lightest CP-even Higgs boson $h^{0}$. In the calculation of the $\gamma \gamma \rightarrow h^{0} h^{0}$ cross section in the decoupling limit, we replace the $\lambda_{h^{0} h^{0} h^{0}}^{(0)}$ coupling by its effective coupling given in Eq. (27) which corresponds, in this limit, to an effective 2-loop 2HDM contribution (see Ref. [56] for a detailed discussion).
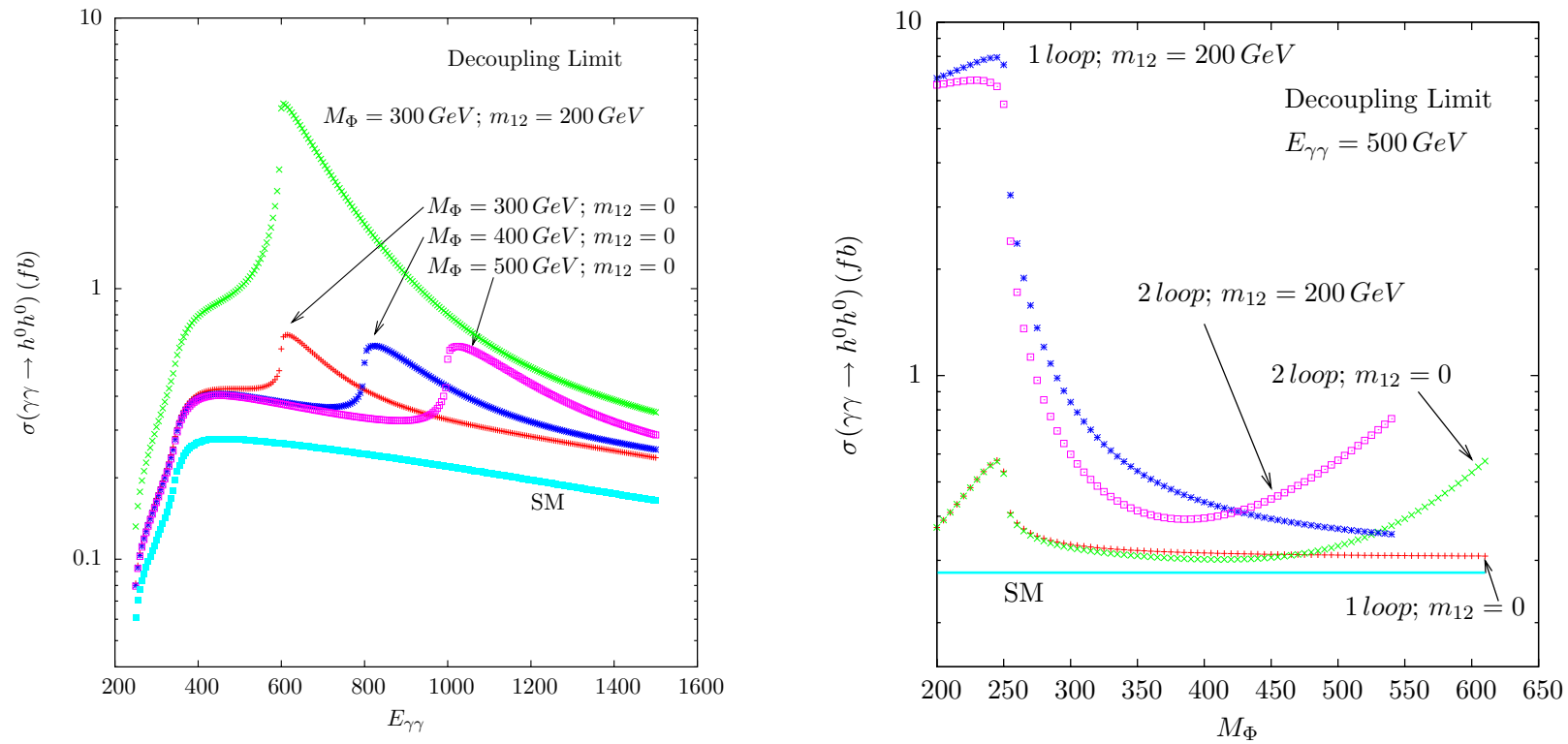

FIG. 11: Cross sections for $h^{0} h^{0}$ production in the decoupling limit with unpolarized photons. On the right we show the loop contributions to the total cross section as a function of $m_{\Phi}$ and for two values of $m_{12}, 0$ and $200 \mathrm{GeV}$. On the left panel the cross section as a function $E_{\gamma \gamma}$ is shown for different values of $m_{\Phi}$ and $m_{12}$. The light Higgs mass is $m_{h^{0}}=120 \mathrm{GeV}$.

As stated in the introduction, this process was studied in detail in Refs. [14, 15] in the decoupling limit. In fact, there is a one to one correspondence between our potential and 
the potential used in [14] which relate $\lambda_{5}$ parameter of Ref [14] to our $m_{12}$ by:

$$
m_{12}^{2}=-\lambda_{5} v_{1} v_{2}
$$

From this relation, one can see that $\lambda_{5}<0\left(\right.$ resp $\left.\lambda_{5}>0\right)$ correspond to our $m_{12}^{2}>0$ (resp $m_{12}^{2}<0$ ). In [14], the non-decoupling effects have their origin in the $h^{0} H^{+} H^{-}$and $h^{0} h^{0} H^{+} H^{-}$vertex and are present already at tree level. In their notation, when $\lambda_{5}=0$ $\left(m_{12}=0\right.$ in our notation) and at the same time the charged Higgs mass and the lightest Higgs mass are of the same order we get $\lambda_{h^{0} H^{+} H^{-}} \approx \lambda_{h^{0} h^{0} h^{0}}^{S M}$. Hence, in this limit the 2HDM cross section is very similar to its SM counterpart. As $m_{12}^{2}$ falls to negative values, the cross section drops due to a cancelation between the $m_{12}^{2}$ and the $m_{H^{ \pm}}^{2}$ contributions until the $m_{12}^{2}$ term starts to dominate and the cross section increase again. For positive $m_{12}^{2}$ the cross section is enhanced relative to the SM one and the non-decoupling effects can be quite large as shown in [14] for $\lambda_{5}<0$. Therefore, at the 1-loop level $m_{12}^{2}$ and the charged Higgs mass are the parameters that regulate the non-decoupling effects. In [15], the authors have studied only the case of $m_{12}=0$ and looked for non-decoupling effects in higher order corrections with origin in the vertex $h^{0} h^{0} h^{0}$ as described earlier. In this case the non-decoupling effects appear mainly for large values of the masses. In this section we will combine both the effects of ref [14] and ref [15] and present the results for the case of the unpolarized photon cross section. We will show that even in this case where the cross sections would be severely reduced, there are still regions where the 2HDM CP even Higgs $h^{0}$ could be disentangled from the SM $h_{S M}$.

In the left panel of Fig. 11 we show the cross section for $\gamma \gamma \rightarrow h^{0} h^{0}$ as a function of $E_{\gamma \gamma}$ for $m_{\Phi}=300,400$ and $500 \mathrm{GeV}$ with $m_{12}=0$ together with the case where $m_{12}=200$ $\mathrm{GeV}$ (note that from now on we will be considering $m_{12}^{2}>0$ ) and $m_{\Phi}=300 \mathrm{GeV}$. In this left panel, the coupling $h^{0} h^{0} h^{0}$ is taken at the tree-level without the higher order correction of Eq.(27). The non-decoupling effects due to the charged Higgs mass can be seen in the vicinity of $E_{\gamma \gamma}=2 m_{\Phi}$ where the cross section is of the order of 0.6 to $0.7 \mathrm{fbarn}$. This effect is enhanced for higher values of $m_{12}$. As shown in the plot, for a charged Higgs mass of 300 $\mathrm{GeV}$ and $m_{12}=200 \mathrm{GeV}$ the cross section can reach 5 fbarn and this value grows with large $m_{12}$. In the right panel of Fig. 11 we display the cross section of $\gamma \gamma \rightarrow h^{0} h^{0}$ as a function $m_{\Phi}$. Here, besides the SM value we plot four different scenarios. The one-loop case with $m_{12}=0$ and $m_{12}=200 \mathrm{GeV}$ and the two-loop case with the higher order corrections given 
in Eq.(27) for the same two values of $m_{12}$. One can see that the cross section enhancement due to the large corrections in $\lambda_{h^{0} h^{0} h^{0}}^{e f}$ take place only for large $m_{\Phi}$ if $m_{12}=0$. As $m_{12}$ grows the cross section grows as described in the left panel, but on top of that we get an extra enhancement due to the higher order corrections. Largest values of the cross section, that can reach 10 fbarn, are attained for the low mass region in $m_{\Phi}$. It is also clear from the right panel that without the higher order corrections of Eq.(27), for large $m_{\Phi}$, the $2 \mathrm{HDM}$ cross section is similar to SM one. On the other hand, once those higher order corrections are included, non decoupling effects arise - the cross section then grows relative to the SM one and can be almost two orders of magnitude above the corresponding SM process depending on the value of $m_{\Phi}$. We finally note that the cut on $m_{\Phi}$ at $610 \mathrm{GeV}$ for $m_{12}=0$ and on $m_{\Phi}$ at $550 \mathrm{GeV}$ for $m_{12}=200 \mathrm{GeV}$ is due to unitarity constraints.

\section{HIGGS SIGNATURES}

We are considering a light CP-even Higgs, that is, with a mass of $120 \mathrm{GeV}$ or less. Assuming that all decay channels with some other Higgs boson in the final state are unaccessible, this particle decays predominantly to $b \bar{b}$ in this mass region. The exception is in the fermiophobic Higgs scenario where it decays to two photons although for a mass of $120 \mathrm{GeV}$ one has already to consider the decay to two $\mathrm{W}$ bosons even if one of the $\mathrm{W}$ is off-shell and strongly virtual. The rate at which it decays to each final state depends on the remaining parameters of the $2 \mathrm{HDM}$ (see [57, 58] for details). The two subleading decays that compete with $h^{0} \rightarrow b \bar{b}$ are $h^{0} \rightarrow c \bar{c}$ and $h^{0} \rightarrow \tau^{+} \tau^{-}$. In model type I the branching fractions to fermions are the SM ones because the coupling dependence cancels. In model type II, the ratio $\Gamma\left(h^{0} \rightarrow b \bar{b}\right) / \Gamma\left(h^{0} \rightarrow \tau^{+} \tau^{-}\right)$is the SM one. On the other hand it is easy to check that for $\tan \beta \geq 1$ the decay $h^{0} \rightarrow b \bar{b}$ is again the dominant one provided we are in a region with moderate values of $\tan \alpha$. For the remaining Yukawa models the situation does not change dramatically. However, if we take as an example the case where $\Phi_{2}$ couples to the quarks and $\Phi_{1}$ couples to the leptons, we obtain the ratio

$$
\frac{\Gamma\left(h^{0} \rightarrow b \bar{b}\right)}{\Gamma\left(h^{0} \rightarrow \tau^{+} \tau^{-}\right)}=\frac{m_{b}^{2}}{m_{\tau}^{2}} \frac{1}{\tan ^{2} \alpha \tan ^{2} \beta}
$$

in the limit $m_{h} \gg m_{q}$. Even for $\tan \alpha=\tan \beta \approx 3$ the ratio becomes almost 100 times smaller than the corresponding SM ratio. Therefore, a detailed study for each model will 
have to take into account the exact branching fractions for each Yukawa version of the 2HDM.

The dominant background to double Higgs production is $\gamma \gamma \rightarrow W^{+} W^{-}$and non-resonant four jet production. The first one can be reduced by imposing a cut on the invariant mass of each pair of b-jets, $M(q \bar{q})$, forcing it to be close to Higgs mass. An efficient b-tagging would further reduce the background by asking that at least three jets be identified as originating from $b$ quarks. A cut on the polar angle would eliminate the non-resonant 4-jet background. Together they would reduce the backgrounds to a level well below the signal. A more detailed analysis can be found in [13]. In the fermiophobic case, the analysis is greatly simplified by the smallness of the four photon production cross section. We just need to avoid very soft photons which can be done with a sensible cut on the photon's transverse momentum.

The process $\gamma \gamma \rightarrow h^{0} H^{0}$ gives rise to very similar signatures. There are in principle two drawbacks: first the phase space is reduced because there is a heavier Higgs in the final state; second we can not reduce the background by asking the two invariant masses from each pair of b-jets to have a similar magnitude. On the other hand, if the channel $H^{0} \rightarrow h^{0} h^{0}$ is open, it can be dominant. This would lead to a very interesting signal of a six b-jet final state. As for $\gamma \gamma \rightarrow h^{0}\left(H^{0}\right) A^{0}$ we concluded that the cross section is much smaller due to the absence of virtual charged Higgs in the loop.

A pseudo-scalar Higgs decays again mainly as $A^{0} \rightarrow b \bar{b}$ and the subleading competing decays are again $A^{0} \rightarrow c \bar{c}$ and $A^{0} \rightarrow \tau^{+} \tau^{-}$. The situation is similar to the CP-even Higgs case - if $\tan \beta \approx 1$ the $b \bar{b}$ channel is always the dominant and well above the others. Once more if we consider the model where one doublet couples to the quarks and the other couples to the leptons, we obtain the ratio

$$
\frac{\Gamma\left(A^{0} \rightarrow b \bar{b}\right)}{\Gamma\left(A^{0} \rightarrow \tau^{+} \tau^{-}\right)}=\frac{m_{b}^{2}}{m_{\tau}^{2}} \frac{1}{\tan ^{2} \beta}
$$

in the limit $m_{h} \gg m_{q}$. In this case, for $\tan \beta \approx 3$ the ratio becomes 10 times smaller than the corresponding SM ratio. Note that a similar situation can occur in the ratio between decays to down and to up quarks. Obviously, this is true as long as the other channels involving other Higgs are closed. The decays to either $h^{0} Z$ or $W^{+} H^{-}$become dominant as soon as they are kinematically allowed. The last two cases could again lead to interesting final states which are easy to detect. As all the cases discussed refer to light Higgs, even if the Higgs to Higgs channel is open the Higgs decay width will in most situation be well 
below the $G e V$ or a few $G e V$ at most.

A final word about the behavior of the cross section with the scattering angle. We have shown that if the $A^{0}$ and $h^{0}$ masses are of the same order, and because in most models the possible final states are very similar, it will be very hard to distinguish a CP-even from a CP-odd state. In fact, even if one changes the polarization of the initial photons, the differential cross section does not distinguish clearly between the two cases except in regions where either the cross sections are too small to be measured or the angle is too small to be probed.

\section{CONCLUSIONS}

We have calculated the total cross section for $\gamma \gamma \rightarrow S_{i} S_{j}, S_{i}=h^{0}, A^{0}, H^{0}$, in the framework of the 2HDM taking into account perturbativity, unitarity as well as vacuum stability constraints on the scalar potential parameters $\lambda$ 's. All available experimental constraints were also taken into account. For the numerical study, we mainly focused on the $h^{0} h^{0}$ and $A^{0} A^{0}$ production modes. We have studied those processes in the general 2HDM, and in two other limiting scenarios: the fermiophobic limit, a scenario where the lightest CP-even Higgs decouples from the fermions and the decoupling scenario where this same Higgs resembles the SM Higgs boson. We have shown that, for both production modes, the most important contribution to $\sigma\left(\gamma \gamma \rightarrow h^{0} h^{0}\right)$ and to $\sigma\left(\gamma \gamma \rightarrow A^{0} A^{0}\right)$ comes from the charged Higgs $H^{ \pm}$diagrams and also from the diagrams with a resonant heavy Higgs that can decay as $H^{0} \rightarrow h^{0} h^{0}$ or $H^{0} \rightarrow A^{0} A^{0}$. Since the cross section is dominated by the charged Higgs virtual exchange, it does not depend on the Yukawa structure of the model.

We have shown that the cross section for $\gamma \gamma \rightarrow h^{0} h^{0}$ can be more than 100 times larger than the corresponding SM one in vast regions of the parameters space. The parameter space will easily be probed for the largest allowed values of $\tan \beta, m_{12}^{2}$ and $|\sin \alpha|$. A light charged Higgs, that is, below the collider center of mass energy, is preferred. A variable energy collider would be a good option to detect the heavy Higgs resonance. We have argued that knowledge of the charged Higgs effects may be crucial to understand the nature of the Higgs bosons if they are eventually found in future experiments at LHC and/or ILC. In the case of $\gamma \gamma \rightarrow h^{0} h^{0}$, we have illustrated that even with a charged Higgs mass of the order of $300 \mathrm{GeV}$, in agreement with $b \rightarrow s \gamma$, the cross section can have a substantial enhancement 
(clearly at least one order of magnitude above the SM). In case of 2HDM type I, where a light charged Higgs is not ruled out from $b \rightarrow s \gamma$ one can have an even larger enhancement which could be 3 order of magnitude above the SM results.

The analysis in [13] shows that the SM Higgs triple coupling could be probed at a linear collider. As described before, their analysis is mainly based on an invariant mass cut, on the identification of at least 3 jets as originating from b-quarks and on a the polar angle cut $\left|\cos \theta_{b}\right|<0.9$. We have showed that the inclusion of the new 2HDM diagrams do not change the angular distribution as the Higgs angular distribution remains almost flat, so that the same cut could be applied. Moreover, in the two Yukawa versions of the model, $B R(h \rightarrow b \bar{b})$ is at least the SM one if not larger. Because the invariant mass cut is the same, the analysis can be applied directly to the 2HDM case. Therefore, when a complete experimental analysis is completed for the SM, it is ready to be used to constraint the 2HDM parameter space. This is one of the major advantages of this study.

Although other regions give rise to higher cross sections, the very interesting case of the 2HDM decoupling limit can also be probed at the photon collider. The importance of the sign of $m_{12}^{2}$ was studied in a more general context. Clearly, positive $m_{12}^{2}$ (in our notation) can lead to large non-decoupling effects. Non-decoupling effects can also appear due to higher order correction to the triple $h^{0} h^{0} h^{0}$ vertex. We have shown that non-decoupling effects will be more easily seen in the low and in the high $m_{\phi}$ regions as in the intermediate mass region the cross section is closer to the SM values.

In the fermiophobic limit this process is complementary to the LEP production process as it grows with $m_{12}$. Most importantly, it can also probe a part of the parameter space that cannot be accessed at hadron colliders. When $m_{12} \approx 0$ the cross section vanishes at hadron colliders [59]. On the contrary, we have shown that in photon-photon collisions the cross section can reach a few fbarn for $m_{12} \approx 0$ and $\tan \beta \approx 5$. The region of low $m_{12}$ in the fermiophobic scenario will most probably not be excluded until we have access to a photon collider.

Regarding the CP-odd Higgs, we have shown that, for the energies considered, only a light $A^{0}$ will be probed at a photon-photon collider. For $m_{A^{0}} \geq 150 \mathrm{GeV}$ the cross section is virtually zero. We have also covered the mixed CP-even modes $h^{0} H^{0}$ and $H^{0} H^{0}$ final state and checked that they can have comparable cross sections to the $h^{0} h^{0}$ mode if $H^{0}$ is not too heavy. For the other modes, $h^{0} A^{0}$ and $H^{0} A^{0}$ we concluded that their cross section is at 
least one order of magnitude smaller than the $h^{0} h^{0}$ and the $A^{0} A^{0}$ one.

As for the final states we have shown that in the two most popular models, 2HDM-I and 2HDM-II, the $b \bar{b}$ final state is the preferred channel as its branching fraction is always at least the SM one. In this case and for not too heavy $h^{0}$ and $A^{0}$ a $4 b$ final state can be searched for. We have showed the such a study for the SM can also be used for the 2HDM. This study can then be complemented with the final states $2 b 2 \tau$ and $4 \tau$. In other models and when other channels are kinematically available, a detailed study has to be performed taking into account all 2HDM parameters.

A last word: there is a well known complementarity between the LHC and the ILC. In this regard, if some of the 2HDM parameters and/or couplings are measured at the LHC and/or ILC experiments such as Higgs masses and the magnitude of the vertices $h^{0} h^{0} h^{0}$ and $H^{0} h^{0} h^{0}$, this information could then be used at $\gamma \gamma$ experiments in order to extract missing parameters and couplings like $h^{0} H^{+} H^{-}$and $H^{0} H^{+} H^{-}$.

\section{ACKNOWLEDGMENTS}

We would like to thank Fernando Cornet for valuable discussions. C.C.H is supported by the National Science Council of R.O.C under Grant \#s: NSC-97-2112-M-006-001-MY3 and R.B is supported by National Cheng Kung University Grant No. HUA 97-03-02-063. R.S. is supported by the FP7 via a Marie Curie Intra European Fellowship, contract number PIEF-GA-2008-221707.

[1] G. Weiglein et al. [LHC/LC Study Group], Phys. Rept. 426 (2006) 47 arXiv:hep-ph/0410364].

[2] P. W. Higgs, Phys. Rev. Lett. 13 (1964) 508. G. S. Guralnik, C. R. Hagen and T. W. B. Kibble, Phys. Rev. Lett. 13, (1964) 585. F. Englert and R. Brout, Phys. Rev. Lett. 13 (1964) 321.

[3] J. Brau et al. [ILC Collaboration], arXiv:0712.1950 [physics.acc-ph]. A. Djouadi, J. Lykken, K. Monig, Y. Okada, M. J. Oreglia and S. Yamashita, "International Linear Collider Reference Design Report Volume 2: PHYSICS AT arXiv:0709.1893 [hep-ph]. N. Phinney, N. Toge and N. Walker, arXiv:0712.2361 [physics.acc-ph]. T. Behnke et al. [ILC Collaboration], arXiv:0712.2356 [physics.ins-det].

[4] V. I. Telnov, Acta Phys. Polon. B 37 (2006) 633 arXiv:physics/0602172]. 
[5] E. Boos et al., Nucl. Instrum. Meth. A 472, 100 (2001) arXiv:hep-ph/0103090.

[6] J. F. Gunion and H. E. Haber, in Research Directions for the Decade, Proceedings of the Summer Study on high energy physics, Snowmass, Colorado, 1990, edited by E. L. Berger (World Scientific, Singapore, 1992), p.469.

[7] J. F. Gunion and H. E. Haber, Phys. Rev. D 48, (1993) 5109.

[8] D. L. Borden, D. A. Bauer and D. O. Caldwell, Phys. Rev. D 48, 4018 (1993); D. L. Borden, V. A. Khoze, W. J. Stirling and J. Ohnemus, Phys. Rev. D 50, 4499 (1994).

[9] M. M. Muhlleitner, M. Kramer, M. Spira and P. M. Zerwas, Phys. Lett. B 508, (2001) 311. M. M. Muhlleitner, arXiv:hep-ph/0008127.

[10] D. M. Asner, J. B. Gronberg and J. F. Gunion, Phys. Rev. D 67, (2003) 035009.

[11] R. N. Hodgkinson, D. Lopez-Val and J. Sola, arXiv:0901.2257 [hep-ph]; A. Arhrib, R. Benbrik and C. W. Chiang, Phys. Rev. D 77, 115013 (2008) arXiv:0802.0319 [hep-ph]]; A. Arhrib, R. Benbrik and C. W. Chiang, AIP Conf. Proc. 1006 (2008) 112; G. Ferrera, J. Guasch, D. Lopez-Val and J. Sola, PoS RADCOR2007, 043 (2007) arXiv:0801.3907 [hep-ph]]; G. Ferrera, J. Guasch, D. Lopez-Val and J. Sola, Phys. Lett. B 659, 297 (2008) arXiv:0707.3162 [hep-ph]]. M. N. Dubinin and A. V. Semenov, Eur. Phys. J. C 28, 223 (2003) arXiv:hep-ph/0206205.

[12] G. V. Jikia and Yu. F. Pirogov, Phys. Lett. B 283 (1992) 135; G. V. Jikia, Nucl. Phys. B 412, 57 (1994).

[13] R. Belusevic and G. Jikia, Phys. Rev. D 70, 073017 (2004) arXiv:hep-ph/0403303.

[14] F. Cornet and W. Hollik, Phys. Lett. B 669, 58 (2008) arXiv:0808.0719 [hep-ph]].

[15] E. Asakawa, D. Harada, S. Kanemura, Y. Okada and K. Tsumura, Phys. Lett. B 672 (2009) 354 arXiv:0809.0094 [hep-ph]].

[16] S. H. Zhu, C. S. Li and C. S. Gao, Phys. Rev. D 58, 015006 (1998) arXiv:hep-ph/9710424.

[17] Y. J. Zhou, W. G. Ma, H. S. Hou, R. Y. Zhang, P. J. Zhou and Y. B. Sun, Phys. Rev. D 68, 093004 (2003) arXiv:hep-ph/0308226.

[18] S. H. Zhu, J. Phys. G 24 (1998) 1703.

[19] G. J. Gounaris and P. I. Porfyriadis, Eur. Phys. J. C 18 (2000) 181 arXiv:hep-ph/0007110.

[20] G. Abbiendi et al. [OPAL Collaboration], Eur. Phys. J. C 40 (2005) 317 arXiv:hep-ex/0408097]. J. Abdallah et al. [DELPHI Collaboration], Eur. Phys. J. C 38, 1 (2004) arXiv:hep-ex/0410017]. S. Schael et al. [ALEPH, DELPHI, L3 and OPAL 
Collaborations], Eur. Phys. J. C 47 (2006) 547.

[21] C. Amsler et al. [Particle Data Group], Phys. Lett. B 667, 1 (2008).

[22] J. F. Gunion, H. E. Haber, G. L. Kane and S. Dawson, "THE HIGGS HUNTER'S GUIDE,"(Addison-Wesley, Reading, 1990).

[23] A. Djouadi, Phys.Rept.459:1-241,2008. arXiv:hep-ph/0503173.

[24] S. L. Glashow and S. Weinberg, Phys. Rev. D 15 (1977) 1958.

[25] V. D. Barger, J. L. Hewett and R. J. N. Phillips, Phys. Rev. D 41 (1990) 3421.

[26] T. P. Cheng and M. Sher, Phys. Rev. D 35 (1987) 3484. D. Atwood, L. Reina and A. Soni, Phys. Rev. D 55 (1997) 3156; R. Diaz, R. Martinez and J. A. Rodriguez, Phys. Rev. D 63 (2001) 095007; A. E. Carcamo, R. Martinez and J. A. Rodriguez, Eur. Phys. J. C 50 (2007) 935.

[27] H. E. Haber, G. L. Kane and T. Sterling, Nucl. Phys. B 161, 493 (1979); J. F. Gunion, R. Vega and J. Wudka, Phys. Rev. D 42, 1673 (1990); J. L. Basdevant, E. L. Berger, D. Dicus, C. Kao and S. Willenbrock, Phys. Lett. B 313, 402 (1993); V. D. Barger, N. G. Deshpande, J. L. Hewett and T. G. Rizzo, P. Bamert and Z. Kunszt, Phys. Lett. B 306, 335 (1993); A. G. Akeroyd, Phys. Lett. B 368, 89 (1996); M. C. Gonzalez-Garcia, S. M. Lietti and S. F. Novaes, Phys. Rev. D 57, 7045 (1998); A. Barroso, L. Brucher and R. Santos, Phys. Rev. D 60, 035005 (1999).

[28] J. F. Gunion and H. E. Haber, Phys. Rev. D 67, 075019 (2003) arXiv:hep-ph/0207010.

[29] A. Arhrib and G. Moultaka, Nucl. Phys. B 558, 3 (1999) arXiv:hep-ph/9808317.

[30] A. Denner, R. J. Guth, W. Hollik and J. H. Kuhn, Z. Phys. C 51, 695 (1991).

[31] A. W. El Kaffas, O. M. Ogreid and P. Osland, arXiv:0709.4203 [hep-ph]. A. Wahab El Kaffas, P. Osland and O. Magne Ogreid, Phys. Rev. D 76, 095001 (2007).

[32] M. Misiak and M. Steinhauser, Nucl. Phys. B764 62 (2007).

[33] A. G. Akeroyd and C. H. Chen, Phys. Rev. D 75, 075004 (2007) arXiv:hep-ph/0701078. C. H. Chen and C. Q. Geng, JHEP 0610, 053 (2006).

[34] K. Ikado et al., Phys. Lett. 97, 251802 (2006);

[35] P. M. Ferreira, R. Santos and A. Barroso, Phys. Lett. B 603 (2004) 219 [Erratum-ibid. B 629 (2005) 114] arXiv:hep-ph/0406231.

[36] N. G. Deshpande and E. Ma, Phys. Rev. D 18 (1978) 2574.

[37] S. Kanemura, T. Kubota and E. Takasugi, Phys. Lett. B 313, 155 (1993) 
arXiv:hep-ph/9303263.

[38] A. G. Akeroyd, A. Arhrib and E. M. Naimi, Phys. Lett. B 490, 119 (2000) arXiv:hep-ph/0006035. A. Arhrib, arXiv:hep-ph/0012353. J. Horejsi and M. Kladiva, Eur. Phys. J. C 46, 81 (2006) arXiv:hep-ph/0510154].

[39] T. Hahn, Comput. Phys. Commun. 140, 418 (2001); T. Hahn, C. Schappacher, Comput. Phys. Commun. 143, 54 (2002); J. Küblbeck, M. Böhm, A. Denner, Comput. Phys. Commun. 60, 165 (1990).

[40] T. Hahn and J. I. Illana, arXiv:0708.3652 [hep-ph]. T. Hahn and J. I. Illana, Nucl. Phys. Proc. Suppl. 160 (2006) 101; T. Hahn, M. Perez-Victoria, Comput. Phys. Commun. 118, 153 (1999); T. Hahn and J. I. Illana, arXiv:0708.3652 [hep-ph]. T. Hahn, Nucl. Phys. Proc. Suppl. 89, 231 (2000).

[41] G. J. van Oldenborgh, Comput. Phys. Commun. 66, 1 (1991); T. Hahn, Acta Phys. Polon. B 30, 3469 (1999).

[42] T. Hahn, Comput. Phys. Commun. 168 (2005) 78 arXiv:hep-ph/0404043. T. Hahn, Nucl. Instrum. Meth. A 559 (2006) 273 arXiv:hep-ph/0509016.

[43] B. Badelek et al. [ECFA/DESY Photon Collider Working Group], Int. J. Mod. Phys. A 19 (2004) 5097 arXiv:hep-ex/0108012.

[44] A. F. Zarnecki, Acta Phys. Polon. B 34 (2003) 2741; B. Badelek et al. [ECFA/DESY Photon Collider Working Group], Int. J. Mod. Phys. A 19 (2004) 5097; I. F. Ginzburg and G. L. Kotkin, Eur. Phys. J. C 13 (2000) 295 arXiv:hep-ph/9905462.

[45] A. Heister et al. [ALEPH Collaboration], Phys. Lett. B 544, 16 (2002).

[46] P. Abreu et al. [DELPHI Collaboration], Phys. Lett. B 507, 89 (2001); Eur. Phys. J. C 35, $313(2004)$.

[47] P. Achard et al. [L3 Collaboration], Phys. Lett. B 534, 28 (2002); Phys. Lett. B 568, 191 (2003).

[48] G. Abbiendi et al. [OPAL Collaboration], Phys. Lett. B 544, 44 (2002).

[49] A. Rosca, arXiv:hep-ph/0212038v1 (2002).

[50] B. Abbott et al. [D0 Collaboration], Phys. Rev. Lett. 82, 2244 (1999).

[51] T. Affolder et al. [CDF Collaboration], Phys. Rev. D 64, 092002 (2001).

[52] http://www-cdf.fnal.gov/physics/exotic/r2a/20081031.diphoton_higgs_fermiophobic/

[53] V. M. Abazov et al. [D0 Collaboration], Phys. Rev. Lett. 101 (2008) 051801 [arXiv:0803.1514 
[hep-ex]].

[54] I. F. Ginzburg, M. Krawczyk, P. Osland, Nucl. Instrum. Meth. A 472, (2001) 149.

[55] A. Arhrib, W. Hollik, S. Penaranda and M. Capdequi Peyranere, Phys. Lett. B 579 (2004) 361.

[56] S. Kanemura, S. Kiyoura, Y. Okada, E. Senaha and C. P. Yuan, Phys. Lett. B 558 (2003) 157; S. Kanemura, Y. Okada, E. Senaha and C. P. Yuan, Phys. Rev. D 70 (2004) 115002.

[57] L. Brucher and R. Santos, Eur. Phys. J. C 12, 87 (2000); A. G. Akeroyd, M. A. Diaz and M. A. Rivera, Phys. Rev. D 76, 115012 (2007).

[58] S. Kanemura, S. Moretti, Y. Mukai, R. Santos and K. Yagyu, arXiv:0901.0204 [hep-ph].

[59] A. Arhrib, R. Benbrik, R. B. Guedes and R. Santos, Phys. Rev. D 78 (2008) 075002. 